\title{
Speech Processing
}

\author{
Berlin Chen \\ Department of Computer Science \& Information Engineering \\ National Taiwan Normal University




\section{Course Contents}

- Both the theoretical and practical issues for spoken language processing will be considered

- Technology for Automatic Speech Recognition (ASR) will be further emphasized

- Topics to be covered

- Statistical Modeling Paradigms

- Spoken Language Structure

- Hidden Markov Models

- Speech Signal Analysis and Feature Extraction

- Acoustic and Language Modeling

- Search/Decoding Algorithms

- Systems and Applications

- Keyword Spotting, Dictation, Speaker Recognition, Spoken Dialogue, Speech-based Information Retrieval, etc. 


\section{Textbook and References (1/3)}

- References books

- X. Huang, A. Acero, H. Hon. Spoken Language Processing, Prentice Hall, 2001

- Jacob Benesty (ed.), M. Mohan Sondhi (ed.), Yiteng Huang (ed.), Springer Handbook of Speech Processing, Springer, 2007

- M.J.F. Gales and S.J. Young. The Application of Hidden Markov Models in Speech Recognition. Foundations and Trends in Signal Processing, 2008

- C. Manning and H. Schutze. Foundations of Statistical Natural Language Processing. MIT Press, 1999

- T. F. Quatieri. Discrete-Time Speech Signal Processing - Principles and Practice. Prentice Hall, 2002

- J. R. Deller, J. H. L. Hansen, J. G. Proakis. Discrete-Time Processing of Speech Signals. IEEE Press, 2000

- F. Jelinek. Statistical Methods for Speech Recognition. MIT Press, 1999

- S. Young et al.. The HTK Book. Version 3.0, 2000 "http://htk.eng.cam.ac.uk"

- L. Rabiner, B.H. Juang. Fundamentals of Speech Recognition. Prentice Hall, 1993

一 王小川教授, 語音訊號處理, 全華圖書 2004 


\section{Textbook and References (2/3)}

- Reference papers

- L. Rabiner, "A Tutorial on Hidden Markov Models and Selected Applications in Speech Recognition," Proceedings of the IEEE, vol. 77, No. 2, February 1989

- A. Dempster, N. Laird, and D. Rubin, "Maximum likelihood from incomplete data via the EM algorithm," J. Royal Star. Soc., Series B, vol. 39, pp. 1-38, 1977

- Jeff A. Bilmes "A Gentle Tutorial of the EM Algorithm and its Application to Parameter Estimation for Gaussian Mixture and Hidden Markov Models," U.C. Berkeley TR-97-021

- J. W. Picone, "Signal modeling techniques in speech recognition," proceedings of the IEEE, September 1993, pp. 1215-1247

- R. Rosenfeld, "Two Decades of Statistical Language Modeling: Where Do We Go from Here?," Proceedings of IEEE, August, 2000

- H. Ney, "Progress in Dynamic Programming Search for LVCSR," Proceedings of the IEEE, August 2000

- H. Hermansky, "Should Recognizers Have Ears?", Speech Communication, 25(1-3), 1998 


\section{Textbook and References (3/3)}

- L.S. Lee and B. Chen, "Spoken document understanding and organization," IEEE Signal Processing Magazine, vol. 22, no. 5, pp. 42-60, Sept. 2005

- M. Gilbert and J. Feng, "Speech and Language Processing over the Web," IEEE Signal Processing Magazine 25 (3), May 2008

- C. Chelba, T.J. Hazen, and M. Saraclar. Retrieval and Browsing of Spoken Content. IEEE Signal Processing Magazine 25 (3), May 2008 


\section{Introduction}

\section{References:}

1. B. H. Juang and S. Furui, "Automatic Recognition and Understanding of Spoken Language - A First Step Toward Natural Human-Machine Communication,"

Proceedings of IEEE, August, 2000

2. I. Marsic, Member, A. Medl, And J. Flanagan, "Natural Communication with Information Systems," Proceedings of IEEE, August, 2000 


\section{Historical Review}

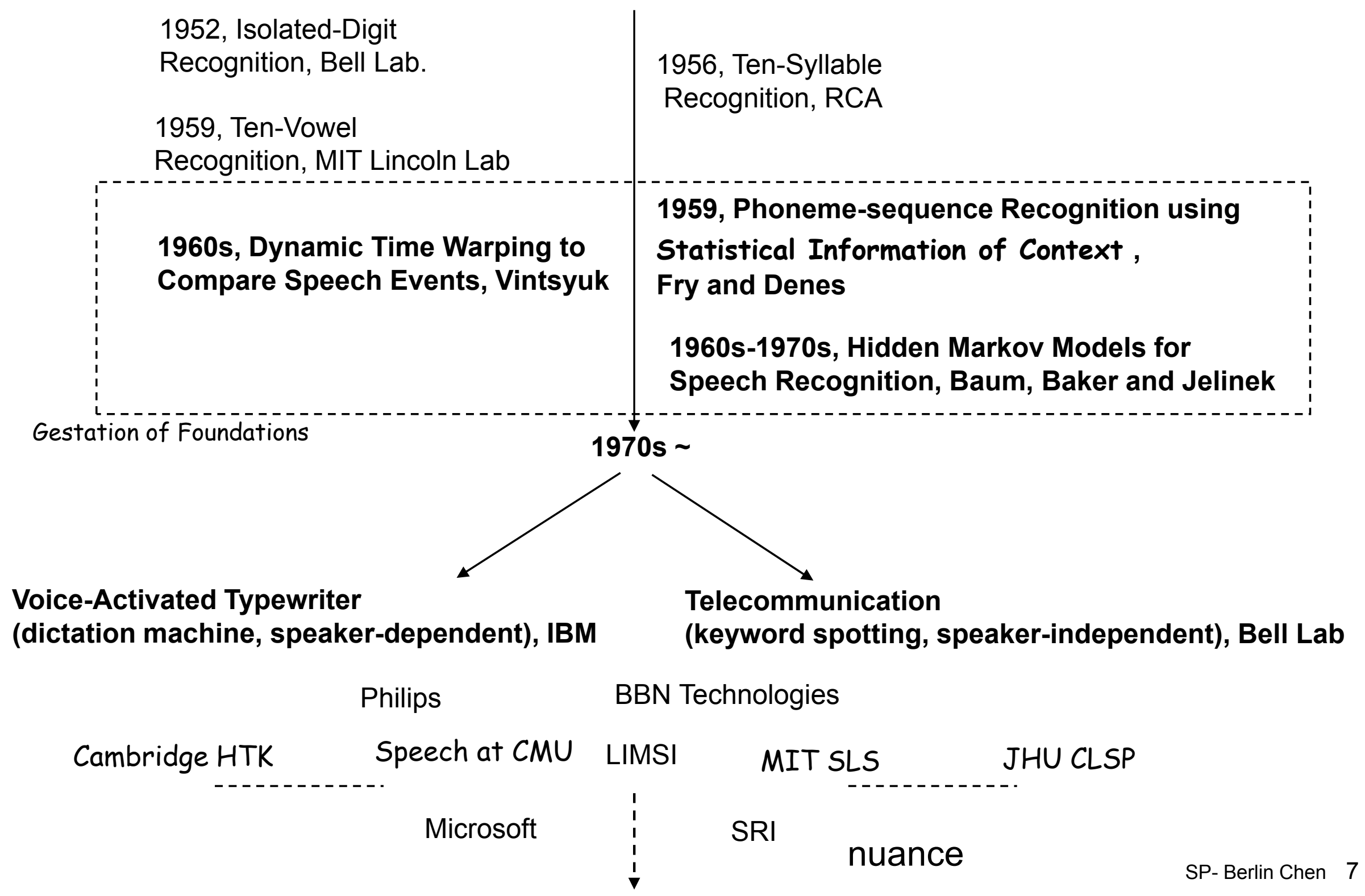




\section{Areas for Speech Processing}

- Production, Perception, and Modeling of Speech

- Signal Processing for Speech

- Speech Coding

- Speech Synthesis (Text-to-Speech)

- Speech Recognition (Speech-to-Text) and Understanding

- Speaker Recognition

- Language Recognition

- Speech Enhancement

- Multichannel Speech Processing 


\section{Progress of Technology (1/6)}

- US. National Institute of Standards and Technology (NIST)

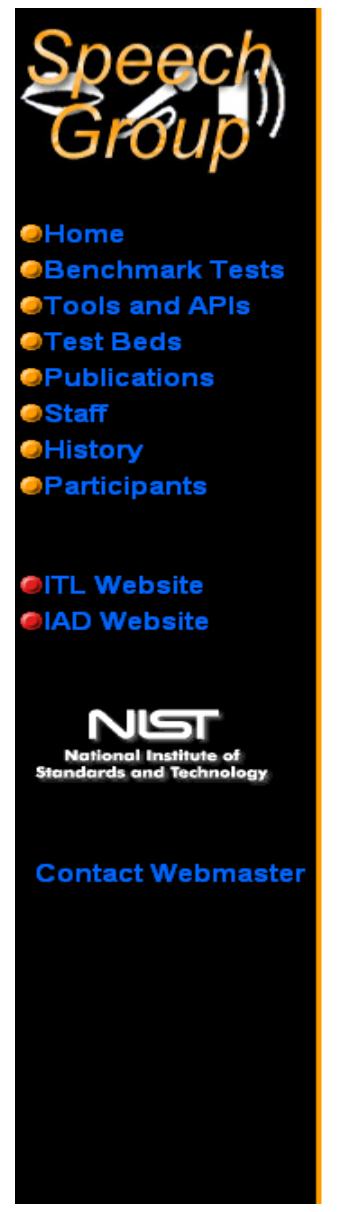

ispeech growp

\section{Mission}

The Speech Group contributes to the advancement of the state-of-the art of spoken language processing (speech recognition and understanding) so that spoken language can reliably serve as an alternative modality for the human-computer interface.

This objective is served by:

- developing measurement methods

- providing materials

nchmark tests within the research and development community

ing prototype systems.

Current Activities

\begin{tabular}{|c|c|c|}
\hline Evaluation & Evaluation Period & Workshop \\
\hline ACE-06 - Automatic Content Extraction & $11 / 06-20 / 06$ & $12 / 14-15 / 06$ \\
\hline $\begin{array}{c}\text { CLEAR-06 - Classification of Locales, Events, } \\
\text { Activities, and Relationships }\end{array}$ & (tbd) & (tbd) \\
\hline GALE-06S - GALE Translation & Jun 22 - Jul 13, 2006 & Sept. 2006 (TBD) \\
\hline LRE-05 - Language Recognition & Oct 24 - Nov 7, 2005 & Dec 6-7, 2005 \\
\hline MT-06 - Machine Translation & July 24 - July 28, 2006 & September '06 (tbd) \\
\hline RT-06S - Rich Transcription Spring Meeting & April 2006 & May 2006 \\
\hline Recognition & April 24 - May 13, 2006 & June 25 - 27, 2006 \\
\hline SRE-06 - Speaker Recognition & November, 2006 & December, 2006 \\
\hline Spoken Term Detection & &
\end{tabular}

http://www.nist.gov/speech/ 


\section{Progress of Technology (2/6)}

- Generic Application Areas (vocabulary vs. speaking style)

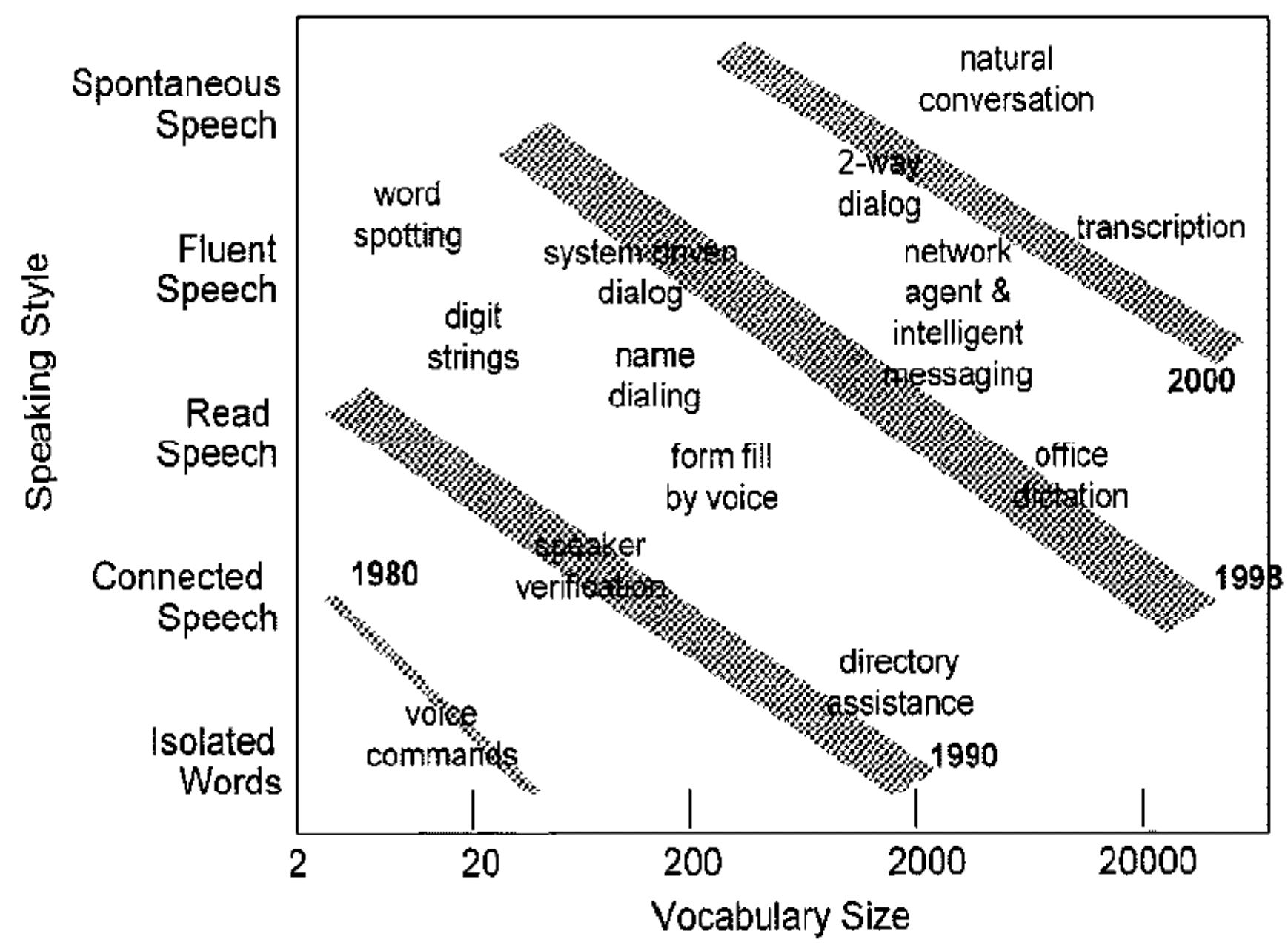




\section{Progress of Technology (3/6)}

- Benchmarks of ASR performance: Overview

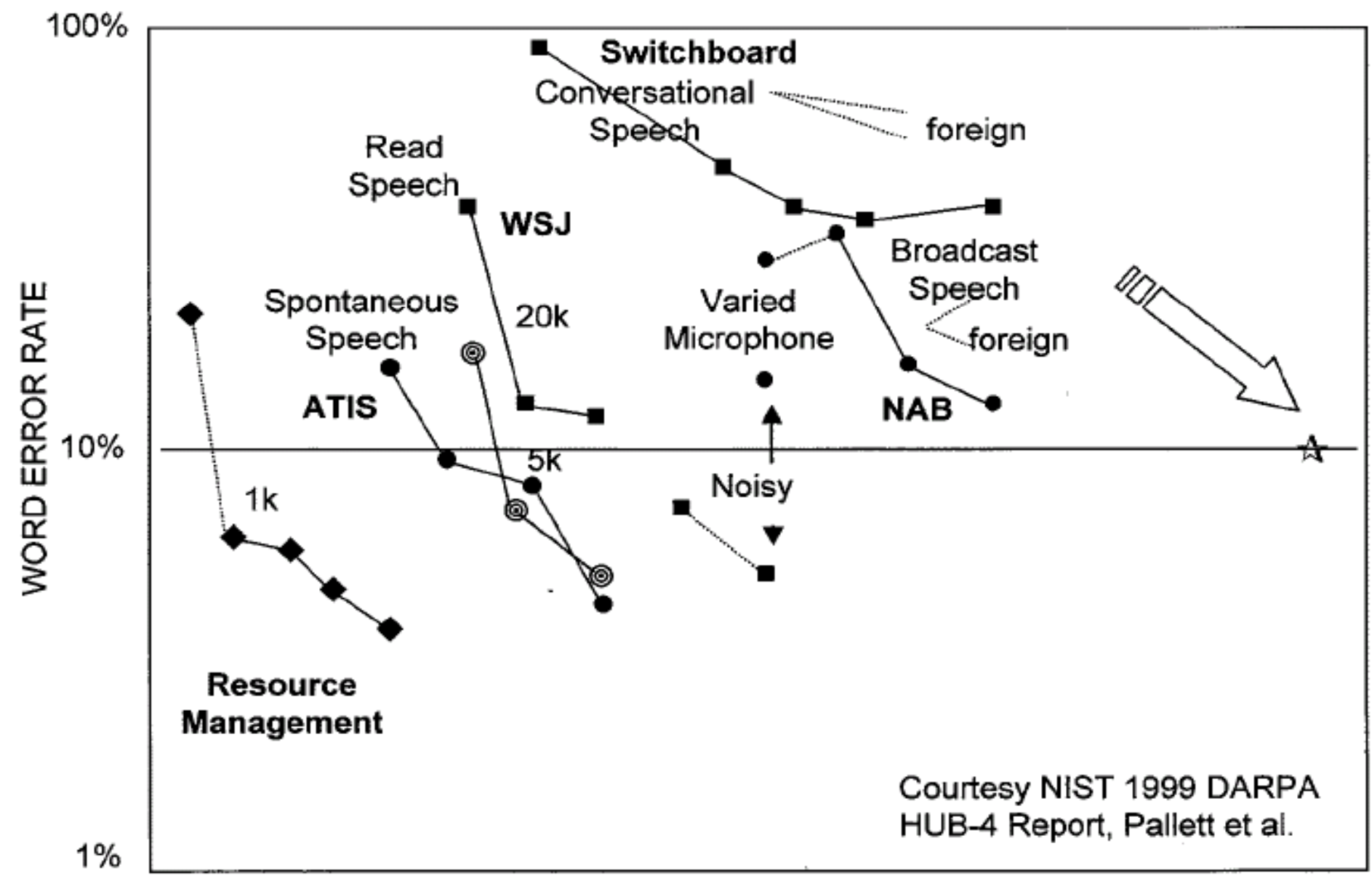

1988198919901991199219931994199519961997199819992000200120022003 


\section{Progress of Technology (4/6)}

- Benchmarks of ASR performance: Broadcast News Speech

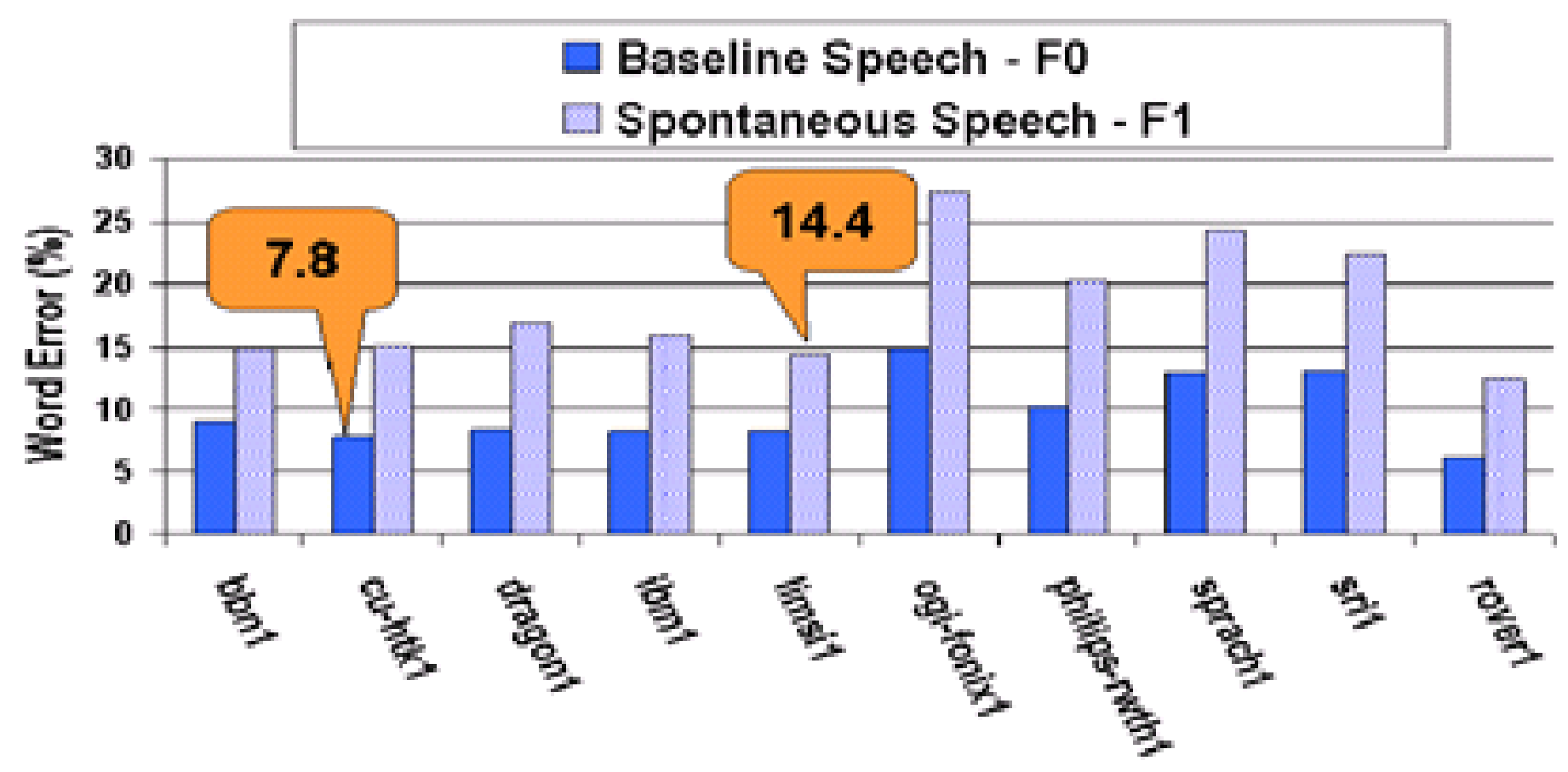




\section{Progress of Technology (5/6)}

- Benchmarks of ASR performance: Conversational Speech
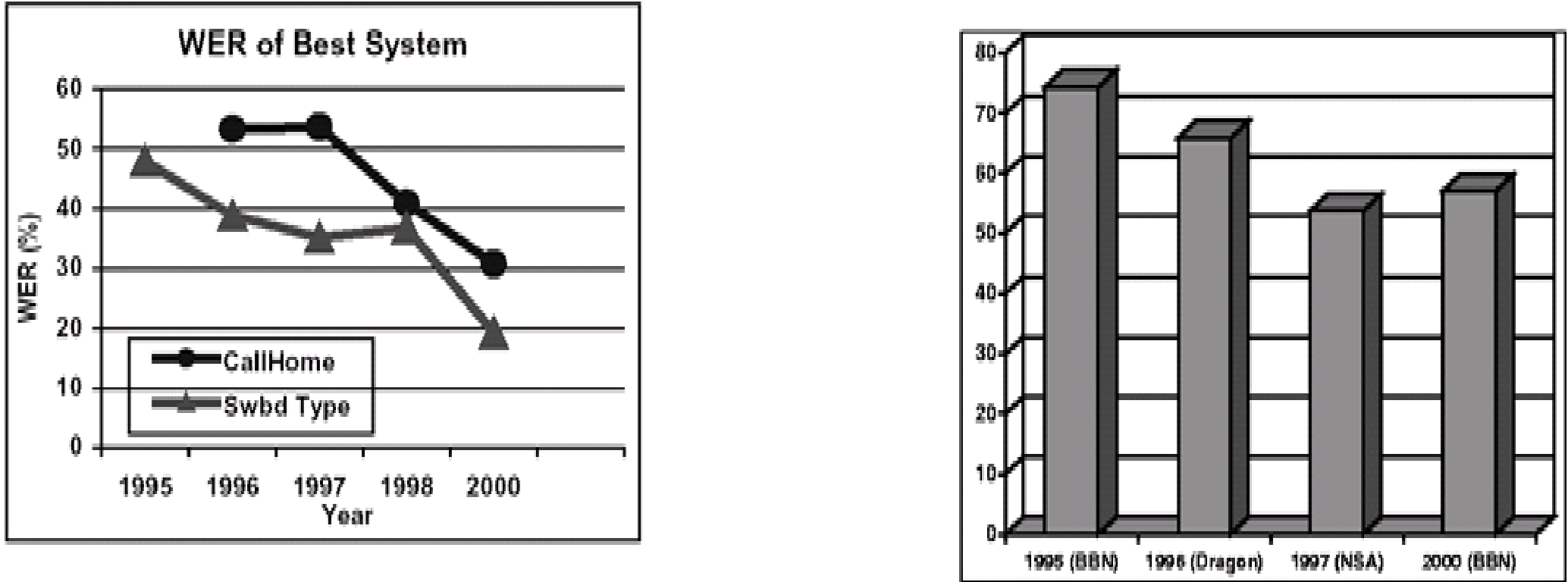

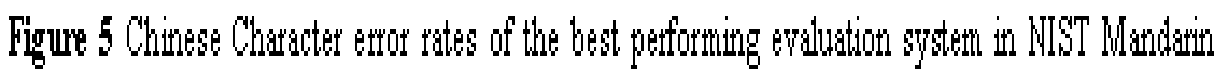

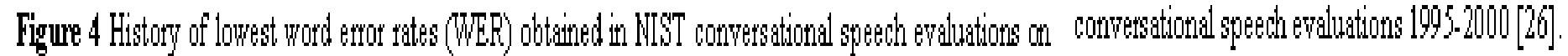

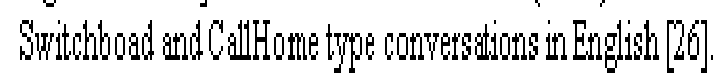




\section{Progress of Technology (6/6)}

- Mandarin Conversational Speech (2003 Evaluation)

- Acoustic/Training Test Data:

- training data: 34.9 hours, 379 sides, from LDC CallHome (22.4hrs) and CallFriend (12.5hrs), 451K Words (+7K English word), 628K Characters

- development data: dev02 1.94 hours from CallFriend

\begin{tabular}{|cc||c|c|}
\hline \multicolumn{1}{|c||}{} & \multicolumn{2}{c|}{ CER (\%) } \\
\cline { 3 - 4 } & & dev02 & eval03 \\
\hline \hline P1 & trans for VTLN & 55.1 & 54.7 \\
\hline P2 & trans for MLLR & 50.8 & 51.3 \\
\hline P3 & lat gen (bg) & 49.3 & 50.5 \\
& tgintcat rescore & 48.9 & 49.8 \\
\hline \hline P4 & lat MLLR & 48.6 & 49.5 \\
\hline \hline CN & P4 & 47.9 & 48.6 \\
\hline
\end{tabular}

- Adopted from 


\section{Determinants of Speech Communication}

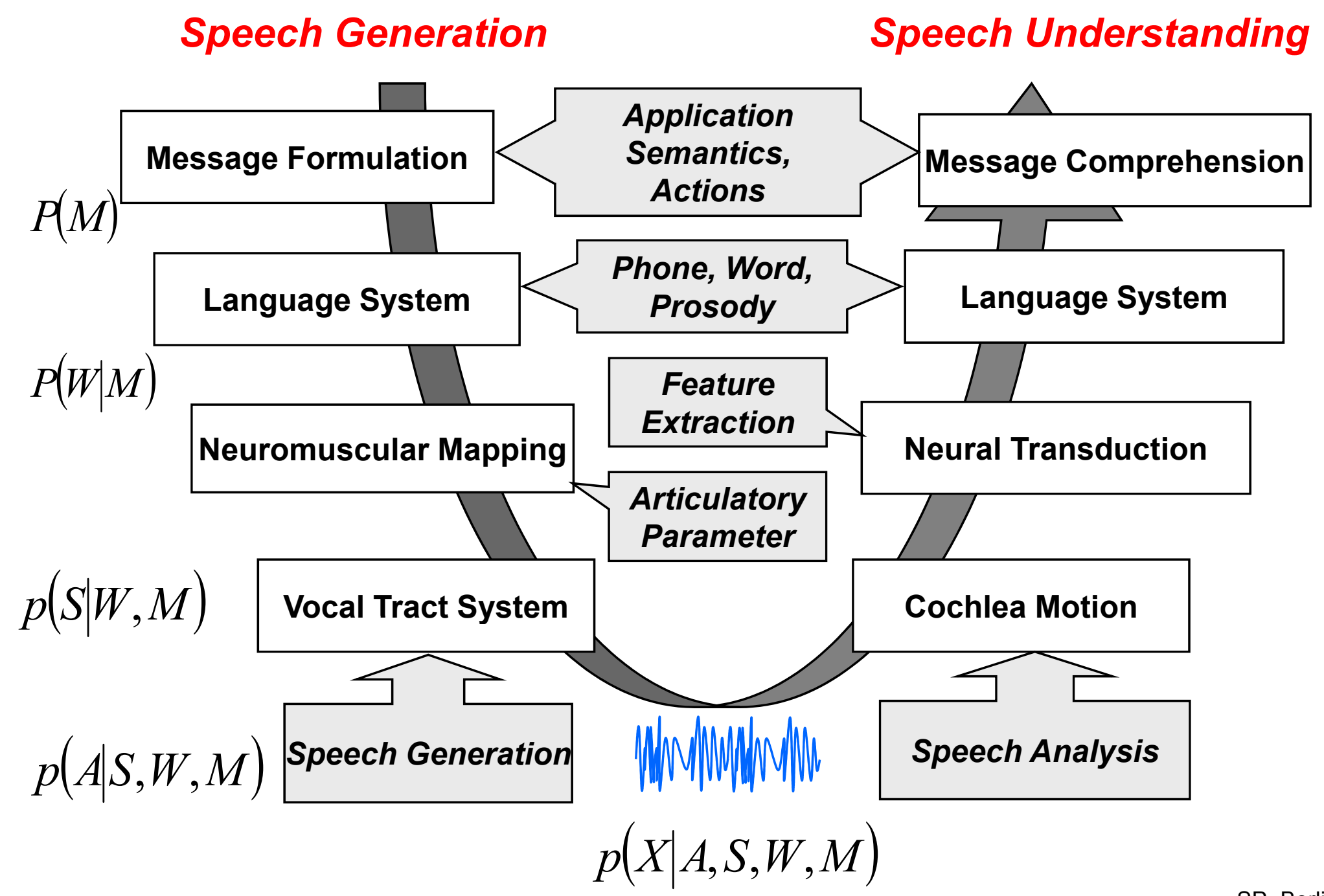




\section{Statistical Modeling Paradigm (1/2)}

- The statistical modeling paradigm used in speech and language processing

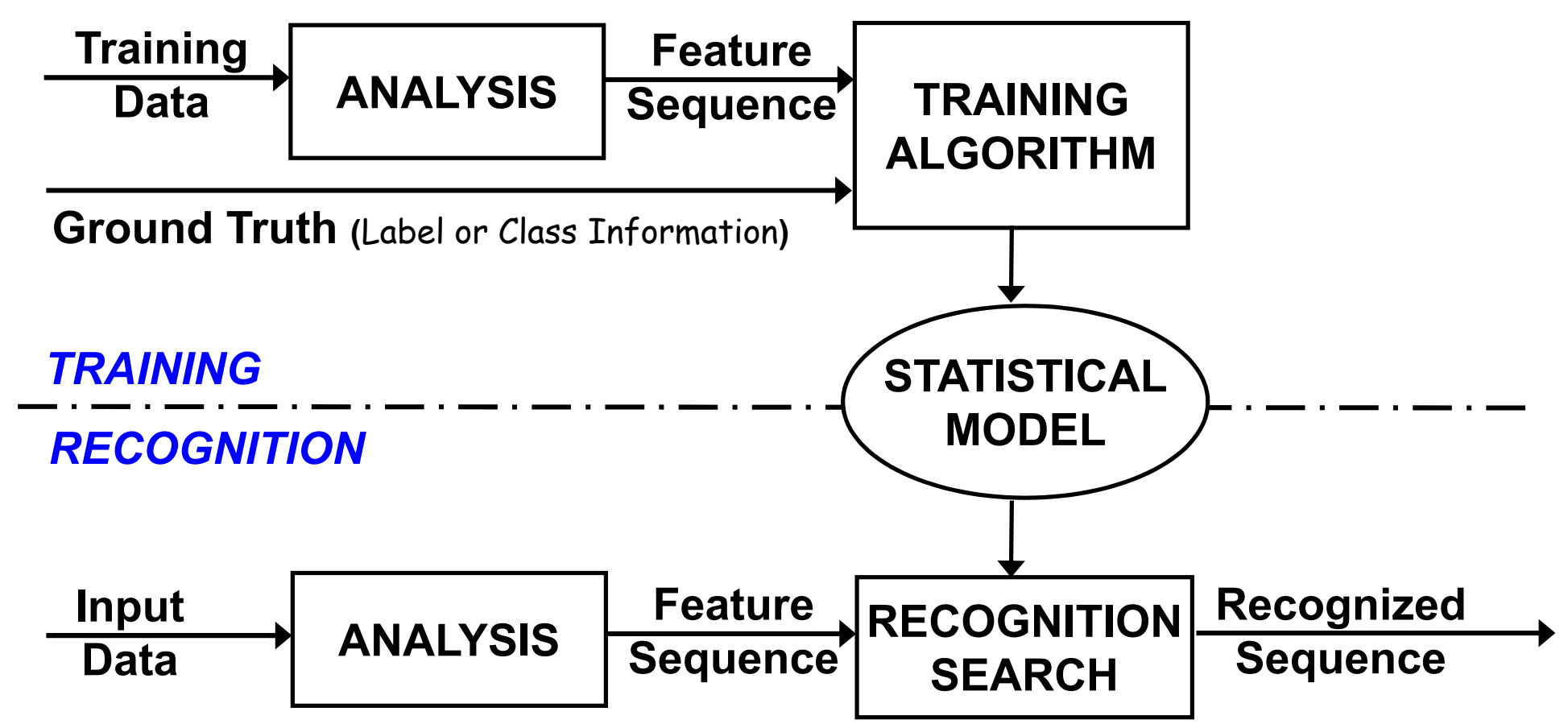




\section{Statistical Modeling Paradigm (2/2)}

- Approaches based on Hidden Markov Models (HMMs) dominate the area of speech recognition

- HMMs are based on rigorous mathematical theory built on several decades of mathematical results developed in other fields

- HMMs are generated by the process of training on a large corpus of real speech data

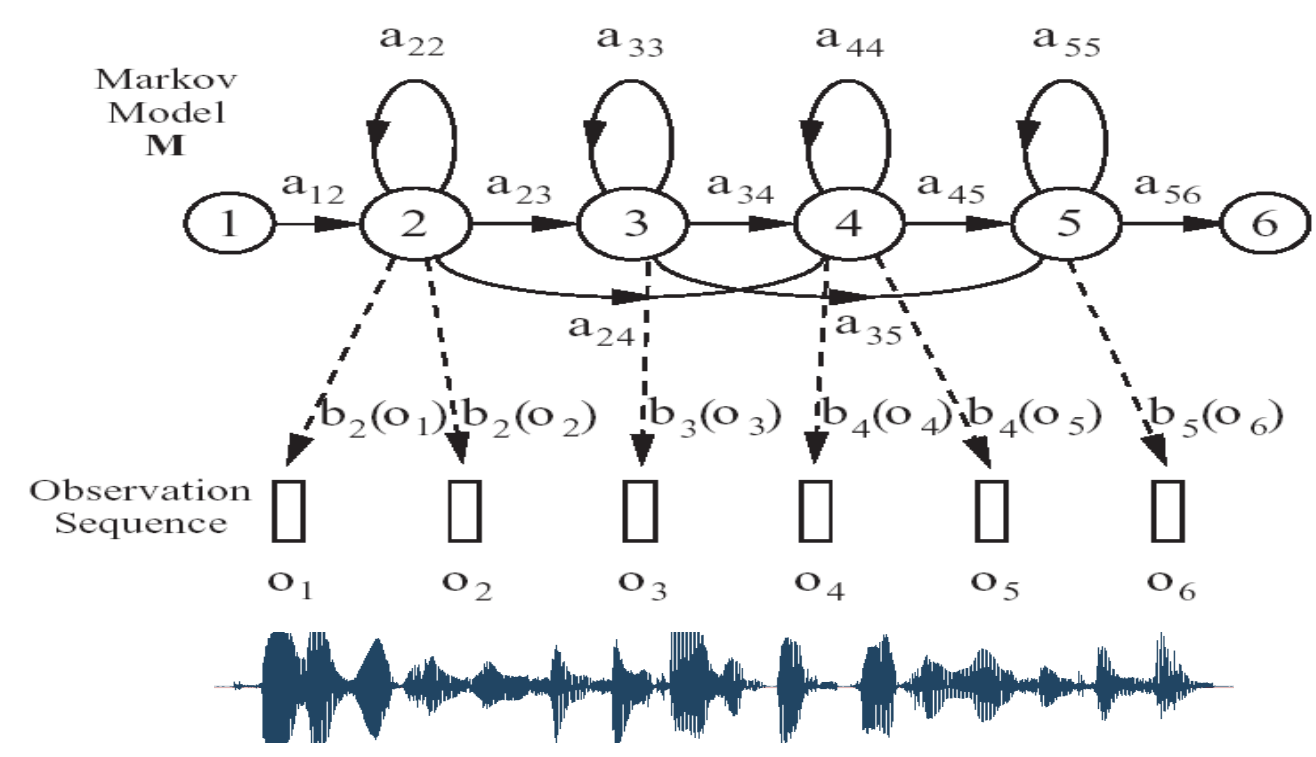




\section{Difficulties: Speech Variability}

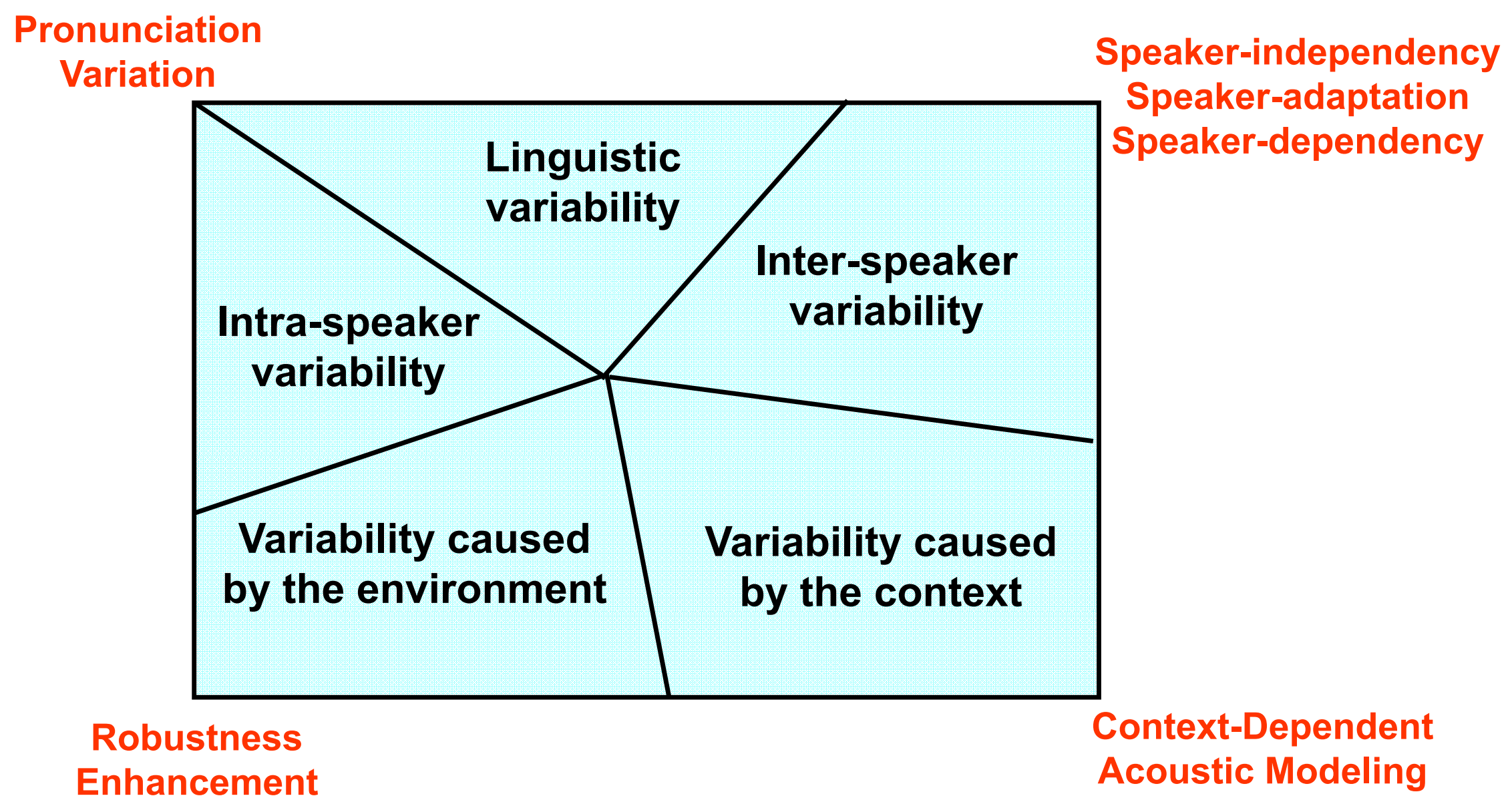




\section{Large Vocabulary Continuous Speech Recognition (LVCSR) (1/3)}

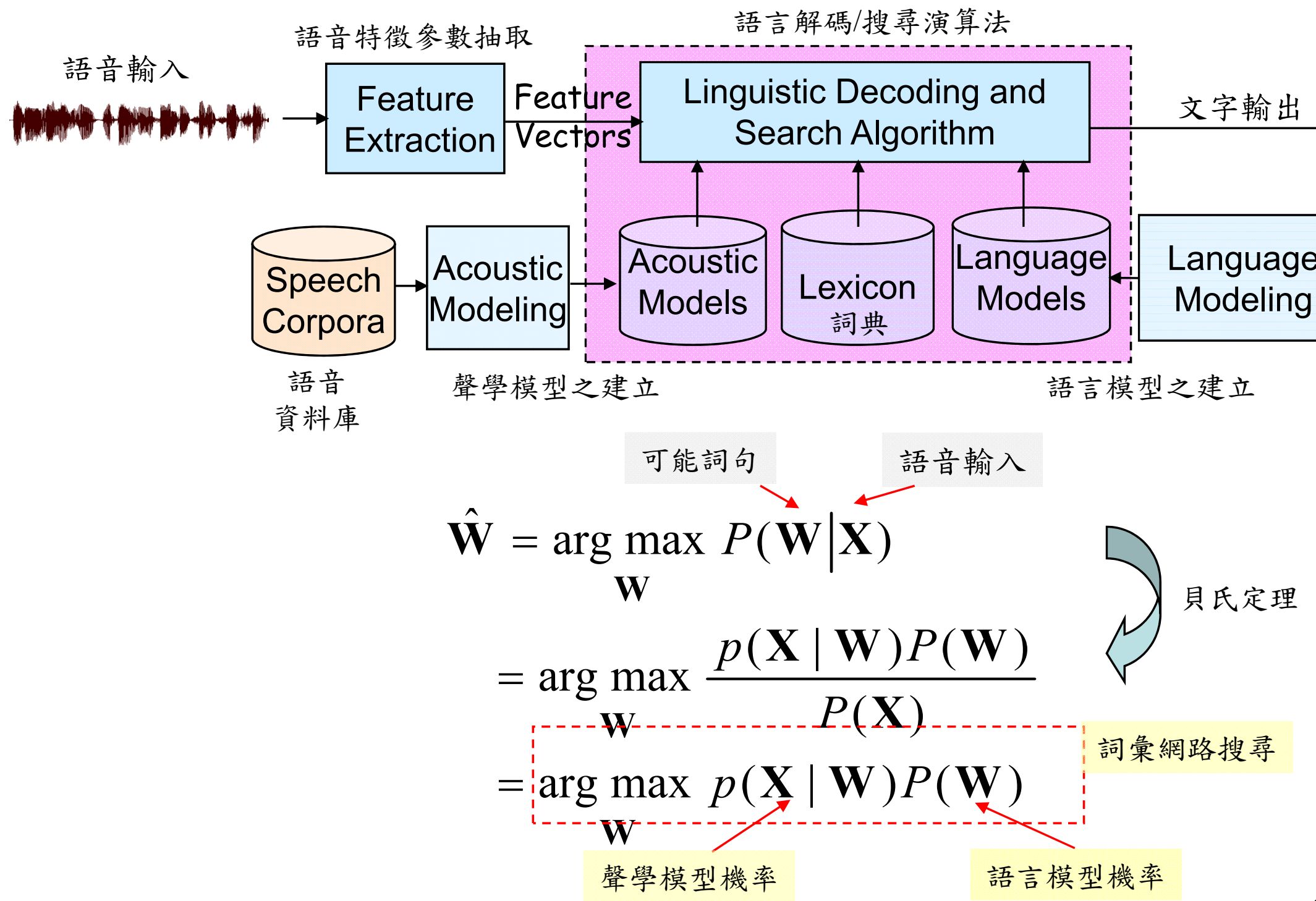




\section{Large Vocabulary Continuous Speech Recognition (cont.) (2/3)}

- Transcription of Broadcast News Speech

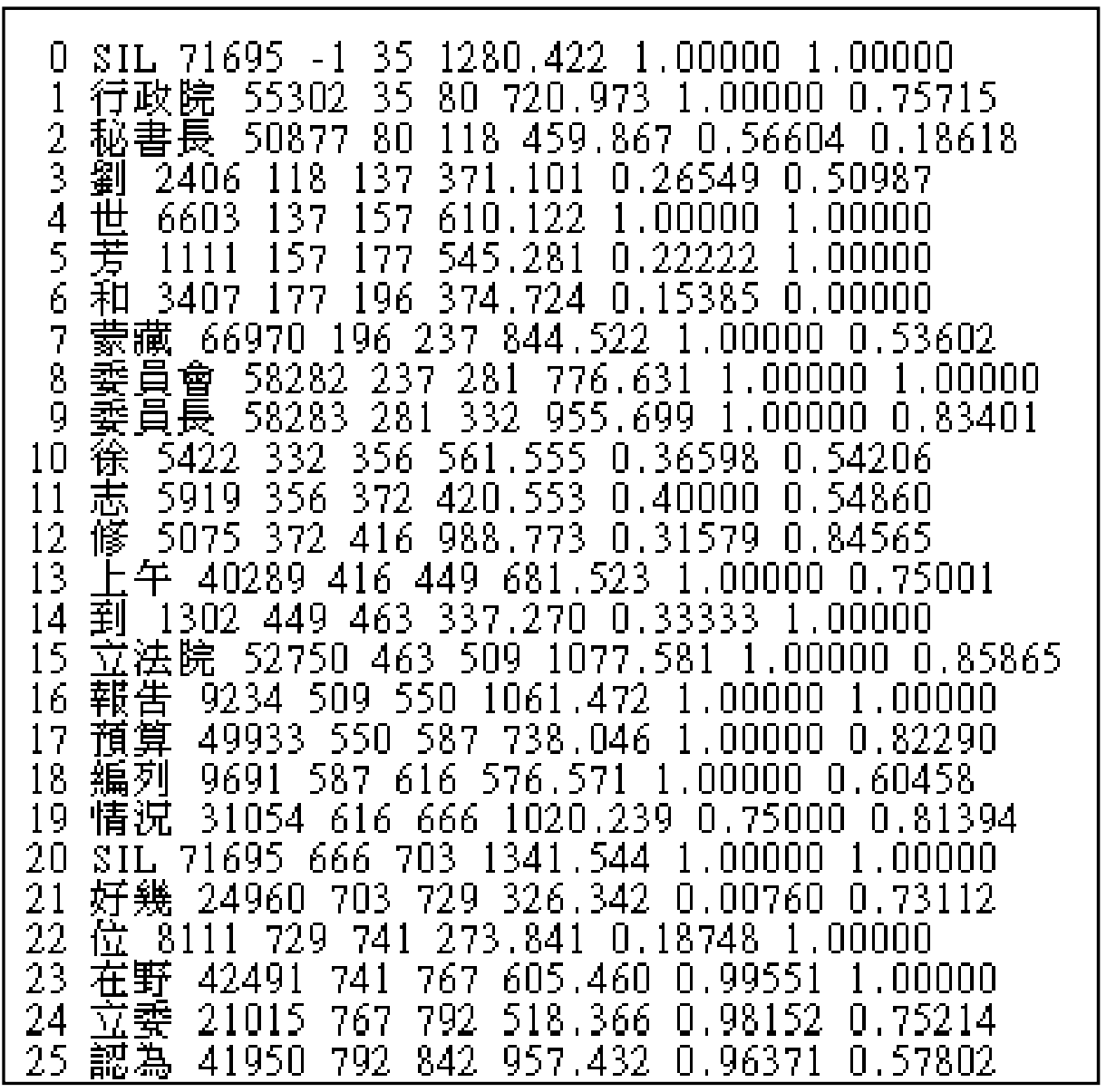

26 SIL $71695 \quad 842872 \quad 1138.477 \quad 1$, 000001 , 00000

27 行政院 $55302872 \quad 934 \quad 1120.105 \quad 0.86107 \quad 0.87346$

28 底外 $29583934971804.2590 .86107 \quad 0.95910$

29 不 $369971988288.728 \quad 0.69917 \quad 1.00000$

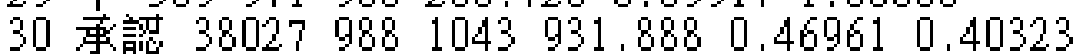

31 外蒙 $47896 \quad 1043 \quad 1084 \quad 786.448 \quad 1$.00000 1 .00000

32 為 $8063 \quad 1084 \quad 1100 \quad 316.677 \quad 0.30057 \quad 1$, 00000

33 我國 $47848 \quad 11001135804.705 \quad 1$.00000 1 .00000

34 領土 $20696 \quad 11351186 \quad 778.006 \quad 0.76186 \quad 0.96218$

35 主非 $36487 \quad 1186 \quad 1237 \quad 1003.320 \quad 0.07122 \quad 1.00000$

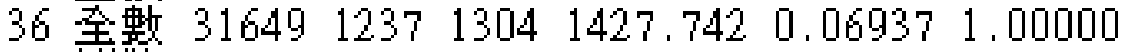

37 刪陸 $39728 \quad 1304 \quad 1349818.702 \quad 1.00000 \quad 0.65401$

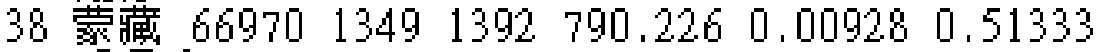

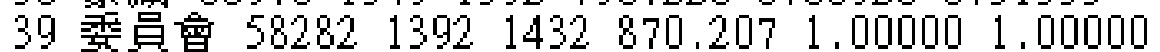

40 的 $1269 \quad 1432 \quad 1441 \quad 165,007 \quad 0.16667 \quad 1$, 00000

41 預算 49933144114901304.0560 .23077 1

42 SIL $71695 \quad 1490 \quad 1522 \quad 1101.7601$.00000 1 .000100

43 猫事 $43981 \quad 152215661100.7800 .055560 .76556$

44 過 $3023 \quad 1566 \quad 1580279.248 \quad 0,07692 \quad 1$ ，00000

45 院镸 $49392 \quad 1580 \quad 1613 \quad 632,123 \quad 0.10656 \quad 0.80456$

46 許 $38001613 \quad 1634526,977 \quad 0.083331$ ，00000

47 志 $5919 \quad 1634 \quad 1650222.692 \quad 0.052631$ 1.00000

$\begin{array}{lllllll}48 & \text { 䧸 } 5420 & 1650 & 1685 & 762.830 & 0.33333 & 0.56287\end{array}$

$\begin{array}{llllllllll}49 & \mathrm{tt} & 7545 & 1685 & 1706 & 484.241 & 0.18462 & 1 & 100000\end{array}$

50 荄 $2847 \quad 1706 \quad 1721 \quad 403.3450 .18182 \quad 1$ ，00000

51 下

52 SIL $71695 \quad 1781 \quad 18432489.8601$.00000 1 .00000 


\section{Large Vocabulary Continuous Speech Recognition (cont.) (3/3)}

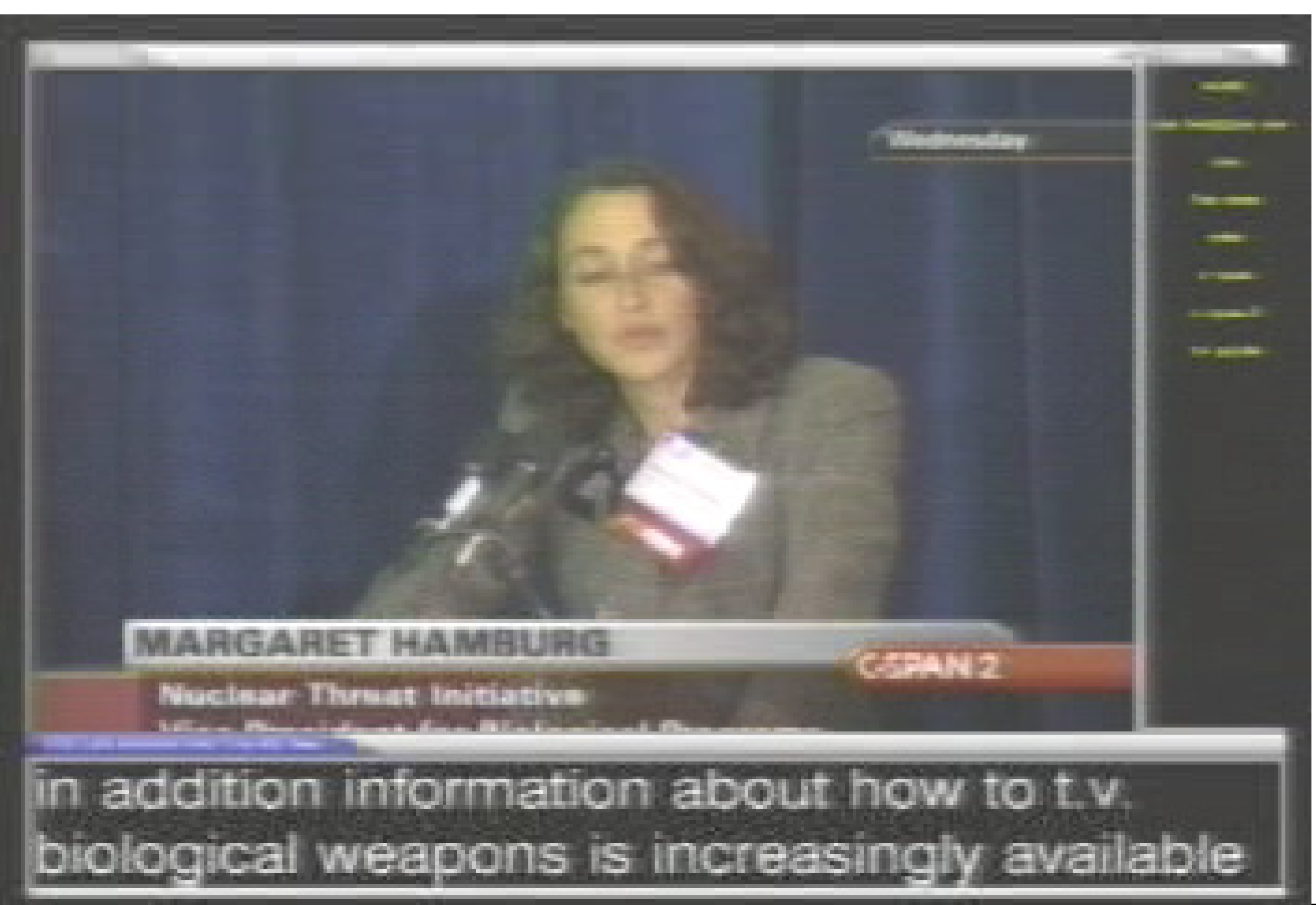




\section{Spoken Dialogue (1/5)}

- Spoken language is attractive because it is the most natural, convenient and inexpensive means of exchanging information for humans

- In mobilizing situations, using keystrokes and mouse clicks could be impractical for rapid information access through small handheld devices like PDAs, cellular phones, etc.

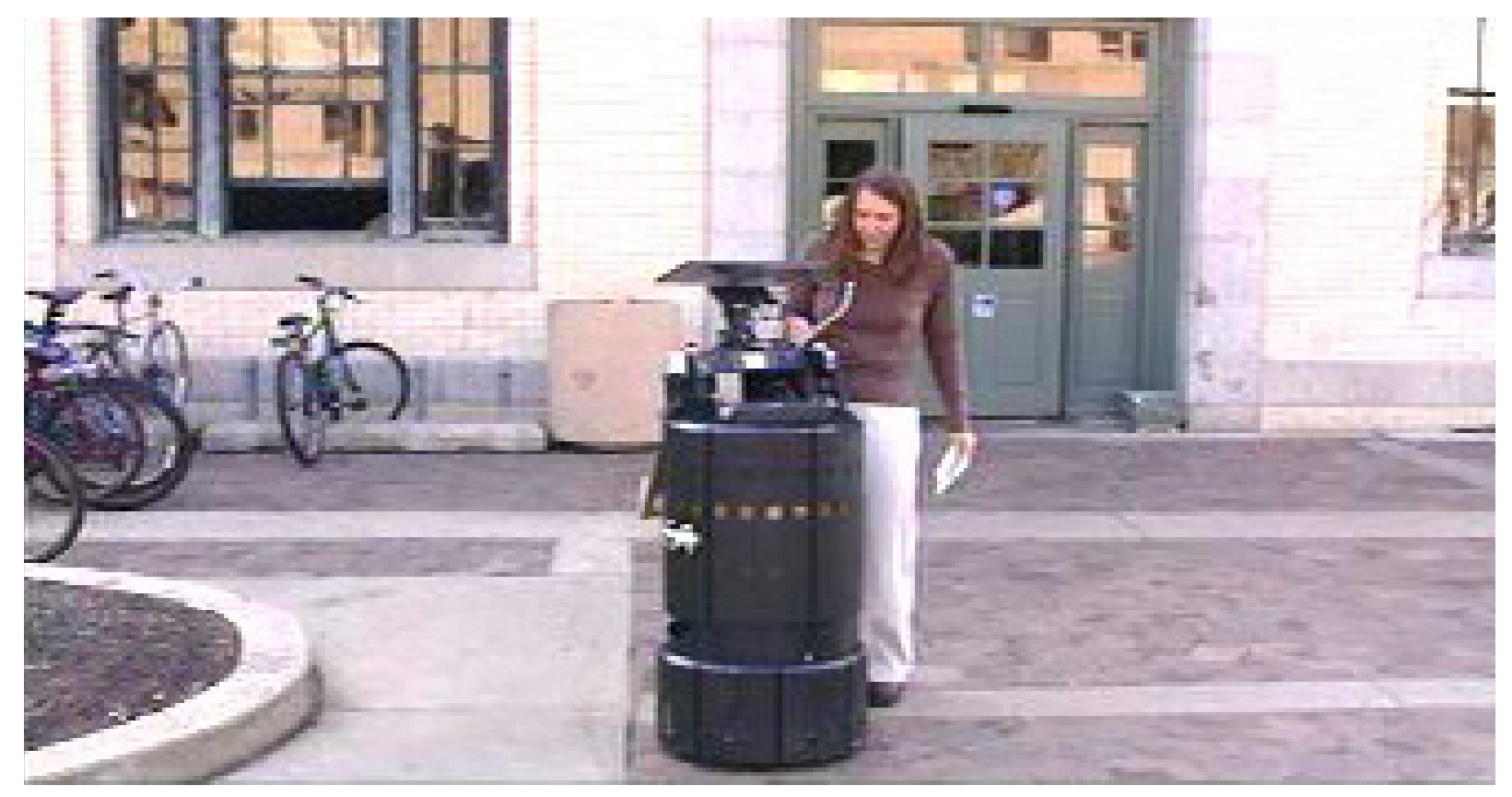




\section{Spoken Dialogue (2/5)}

- Flowchart

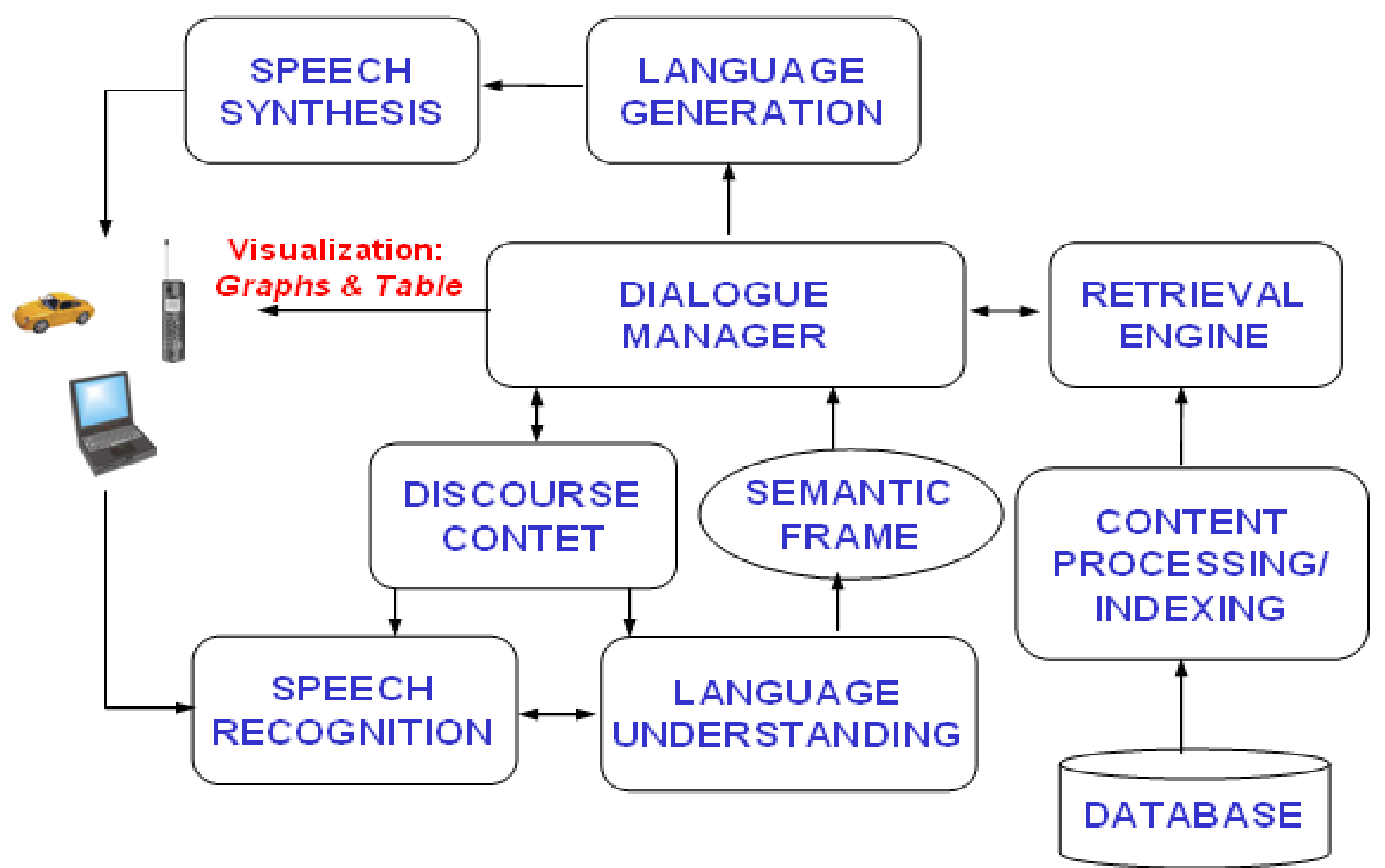

C.f. V. Zue, J.R. Glass, Conversational Interfaces: Advances and Challenges. Proceedings of the IEEE,

Vol. 88, No. 8, August 2000 


\section{Spoken Dialogue (3/5)}

- Multimodality of Input and Output

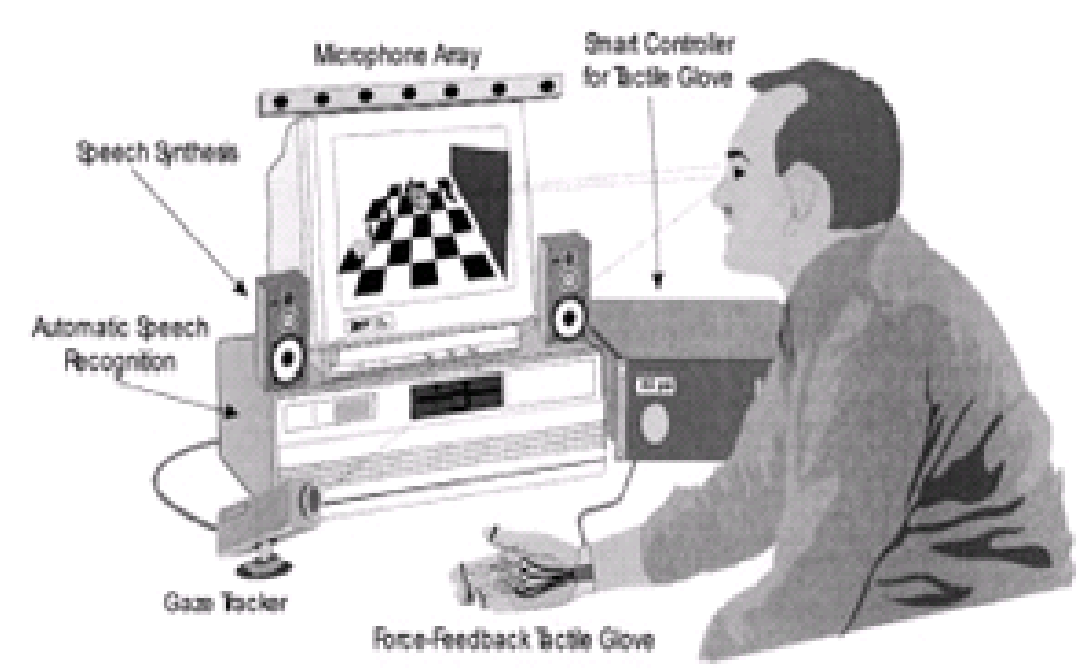

Experimental client workstation incorporating sight, sound, and touch modalities for human/machine communication. The eye tracker provides a gaze-controlled cursor for indicating objects in the display. The tactile force-feedback glove allows displayed objects to be grasped, "felt," and moved. Hands-free speech recognition and synthesis provides natural conversational interaction [7].

C.f. I. Marsic, A. Medl, And J. Flanagan, Natural Communication with Information Systems. Proceedings of the IEEE, 


\section{Spoken Dialogue (4/5)}

- Deployed Dialogue Systems

\begin{tabular}{l||c|c|c|c}
\hline Domain & Language & Vocabulary & \multicolumn{2}{|c}{ Average } \\
& & Size & Words/Utt & Utts/Dialogue \\
\hline CSELT Train Timetable Info & Italian & 760 & 1.6 & 6.6 \\
SpeechWorks Air Travel Reservation & English & 1000 & 1.9 & 10.6 \\
Philips Train Timetable Info & German & 1850 & 2.7 & 7.0 \\
\hline CMU Movie Information & English & 757 & 3.5 & 9.2 \\
CMU Air Travel Reservation & English & 2851 & 3.6 & 12.0 \\
LIMSI Train Timetable Info & French & 1800 & 4.4 & 14.6 \\
MIT Weather Information & English & 1963 & 5.2 & 5.6 \\
MIT Air Travel Reservation & English & 1100 & 5.3 & 14.1 \\
AT\&T Operator Assistance & English & 4000 & 7.0 & 3.0 \\
\hline Air Travel Reservations (human) & English & $?$ & 8.0 & 27.5 \\
\hline
\end{tabular}




\section{Spoken Dialogue (5/5)}

- Topics vs. Dialogue Terms

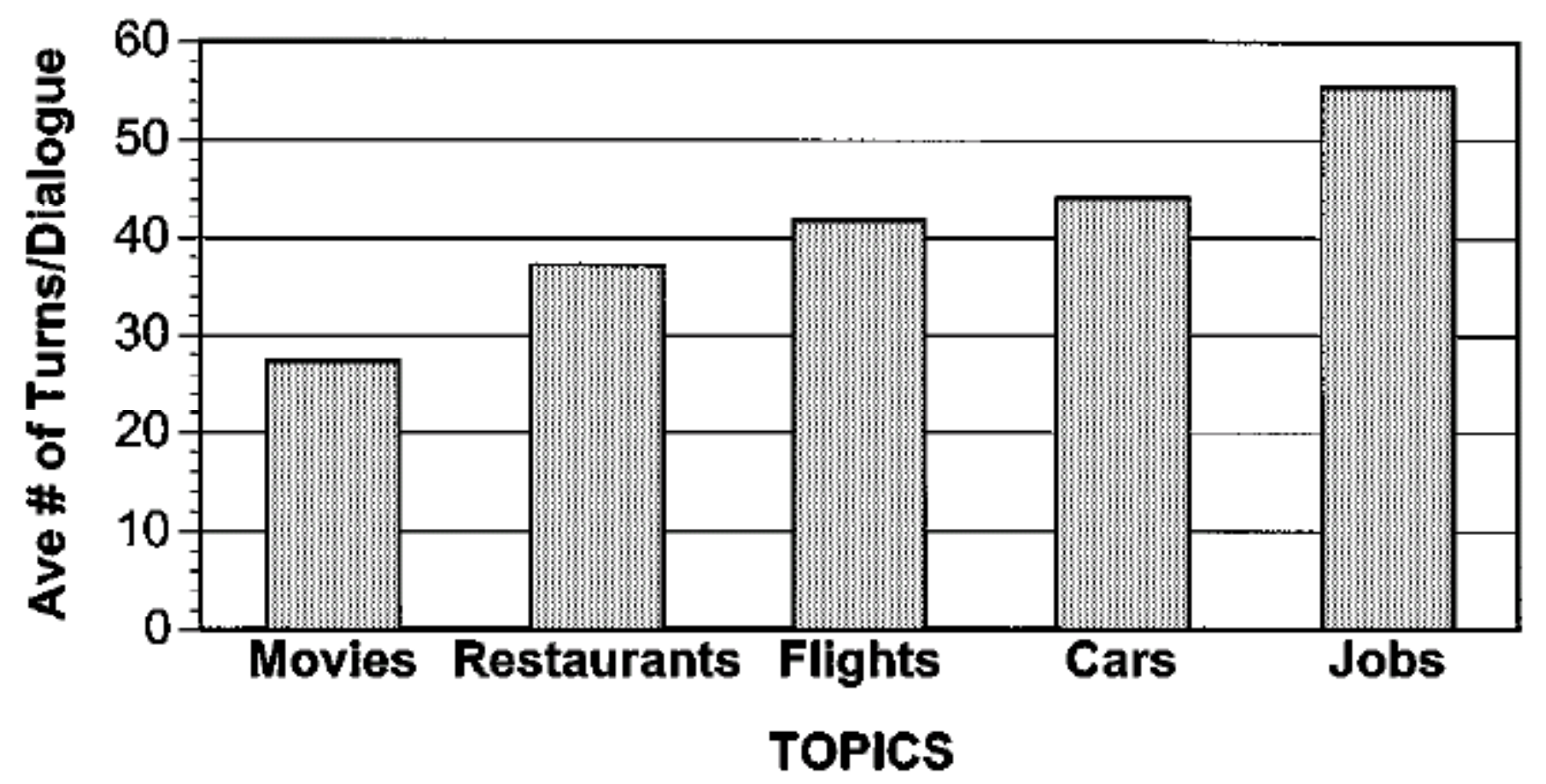




\section{Speech-based Information Retrieval (1/6)}

- Task :

- Automatically indexing a collection of spoken documents with speech recognition techniques

- Retrieving relevant documents in response to a text/speech query

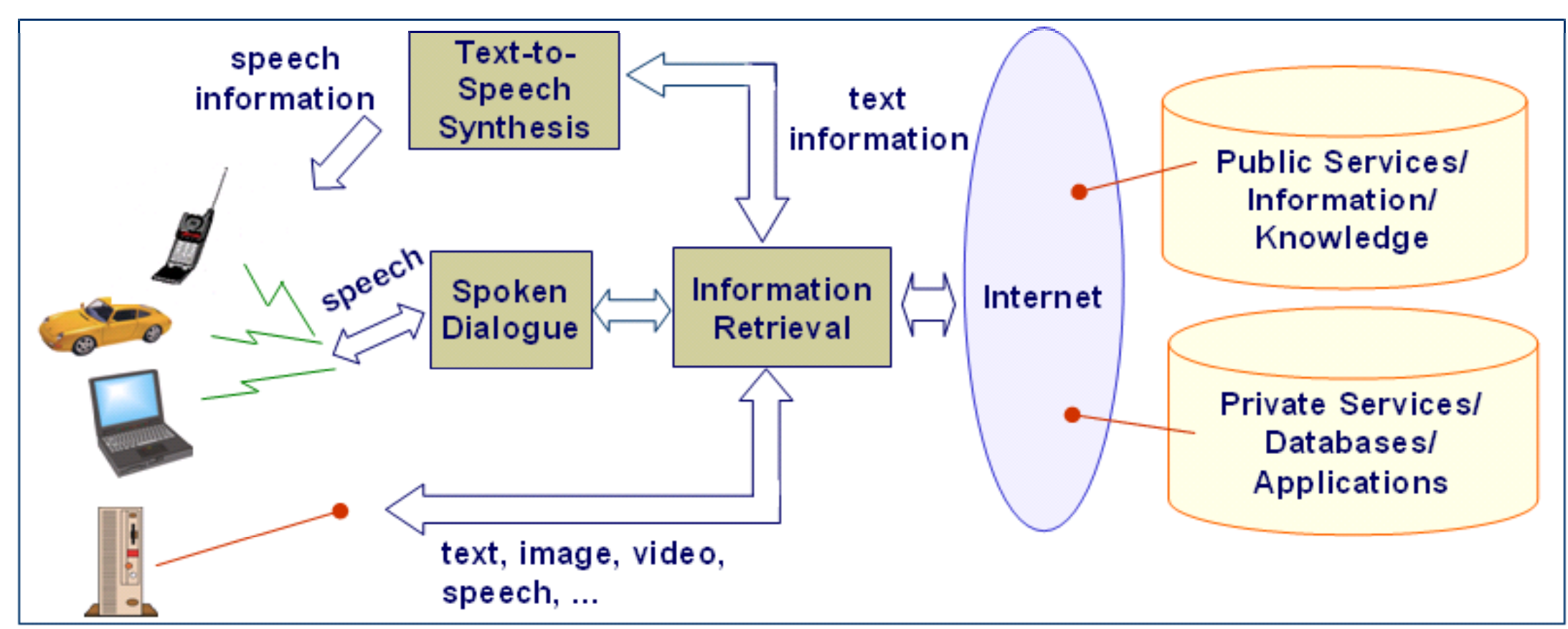




\section{Speech-based Information Retrieval (2/6)}

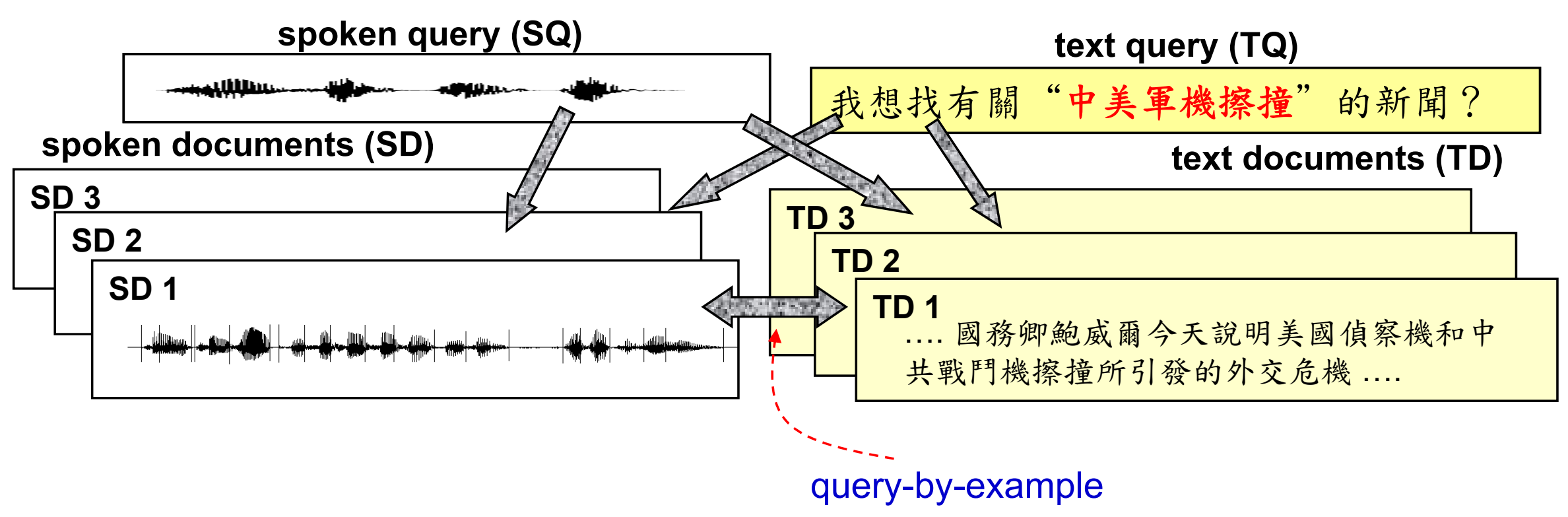

- SQ/SD is the most difficult

- TQ/SD is studied most of the time 


\section{Speech-based Information Retrieval (3/6)}

翰入聲音問句：“請幫我查總統府升旗典䀅”

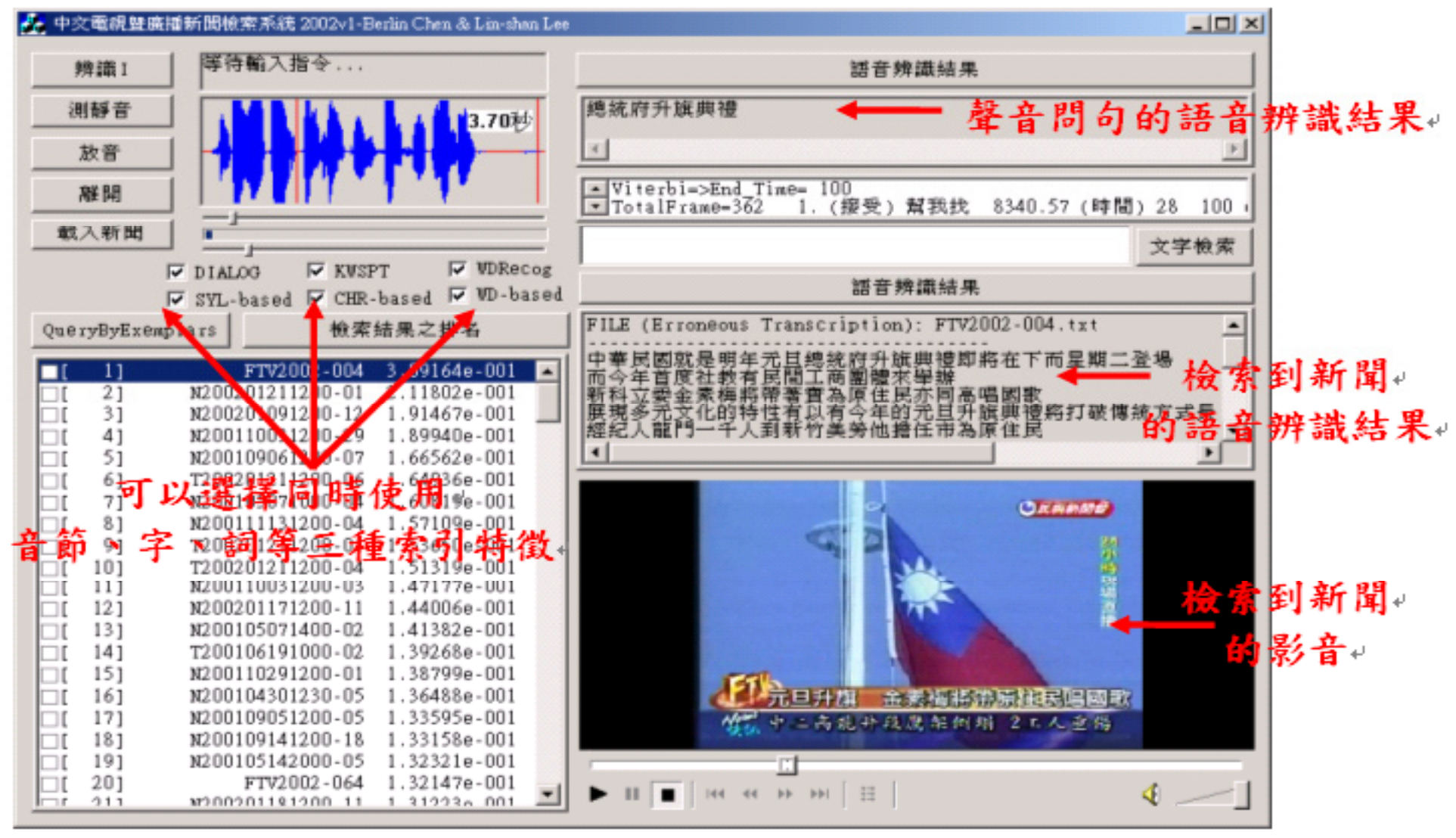

中文語音資訊檢索雛形展示系統。

C.f. B. Chen, H.M. Wang, Lin-shan Lee, "Discriminating capabilities of syllable-based features and approaches of utilizing them for voice retrieval of speech information in Mandarin Chinese", IEEE Transactions on Speech and Audio Processing , Vol. 10, No. 5, pp. 303-314, July 2002. 


\section{Speech-based Information Retrieval (4/6)}

- Spoken queries retrieving text news documents via mobile devices

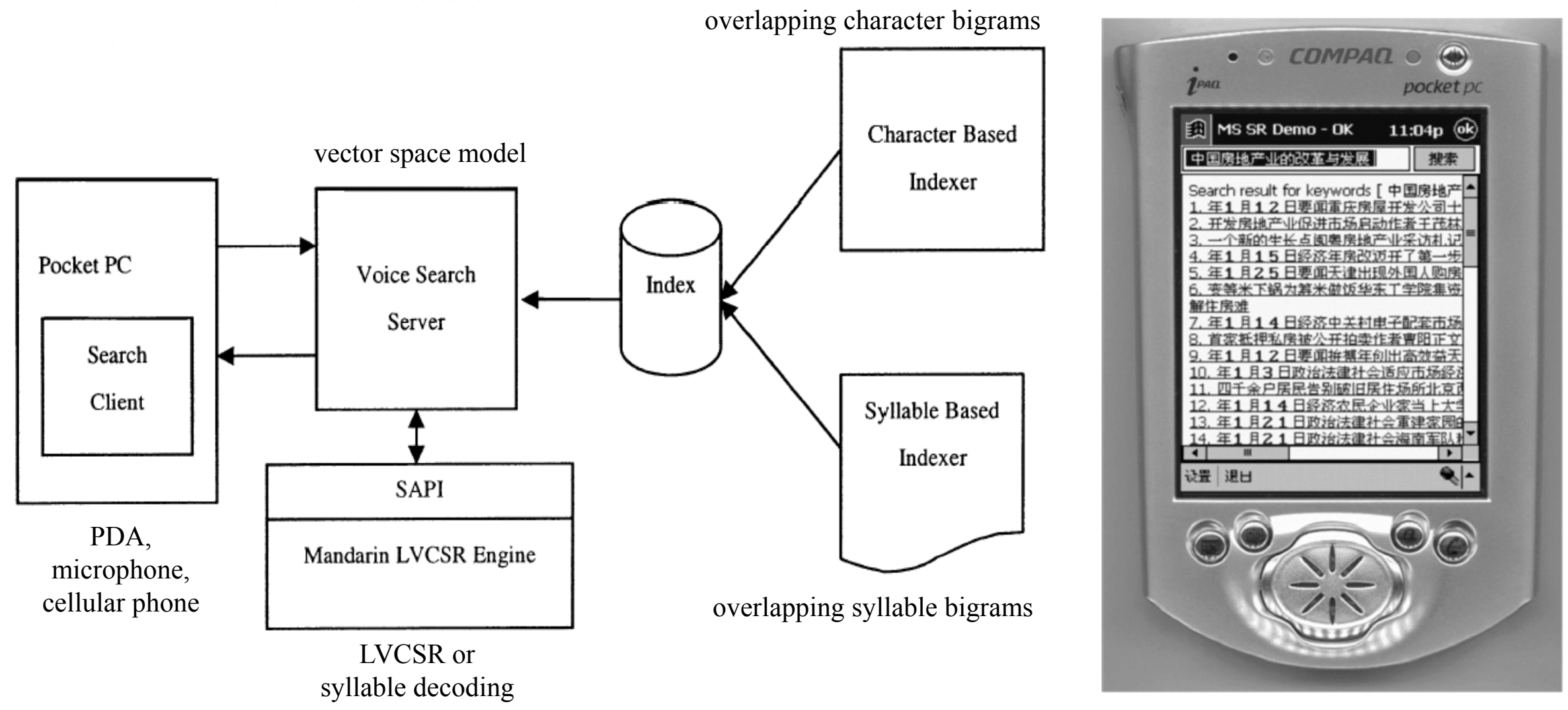

C.f. Chang, E., Seide, F., Meng, H., Chen, Z., Shi, Y., And Li, Y. C. 2002. A system for spoken query

information retrieval on mobile devices. IEEE Trans. on Speech and Audio Processing 10, 8 (2002), 531-541. 


\section{Speech-based Information Retrieval (5/6)}

- Spoken queries retrieving text news documents via mobile devices

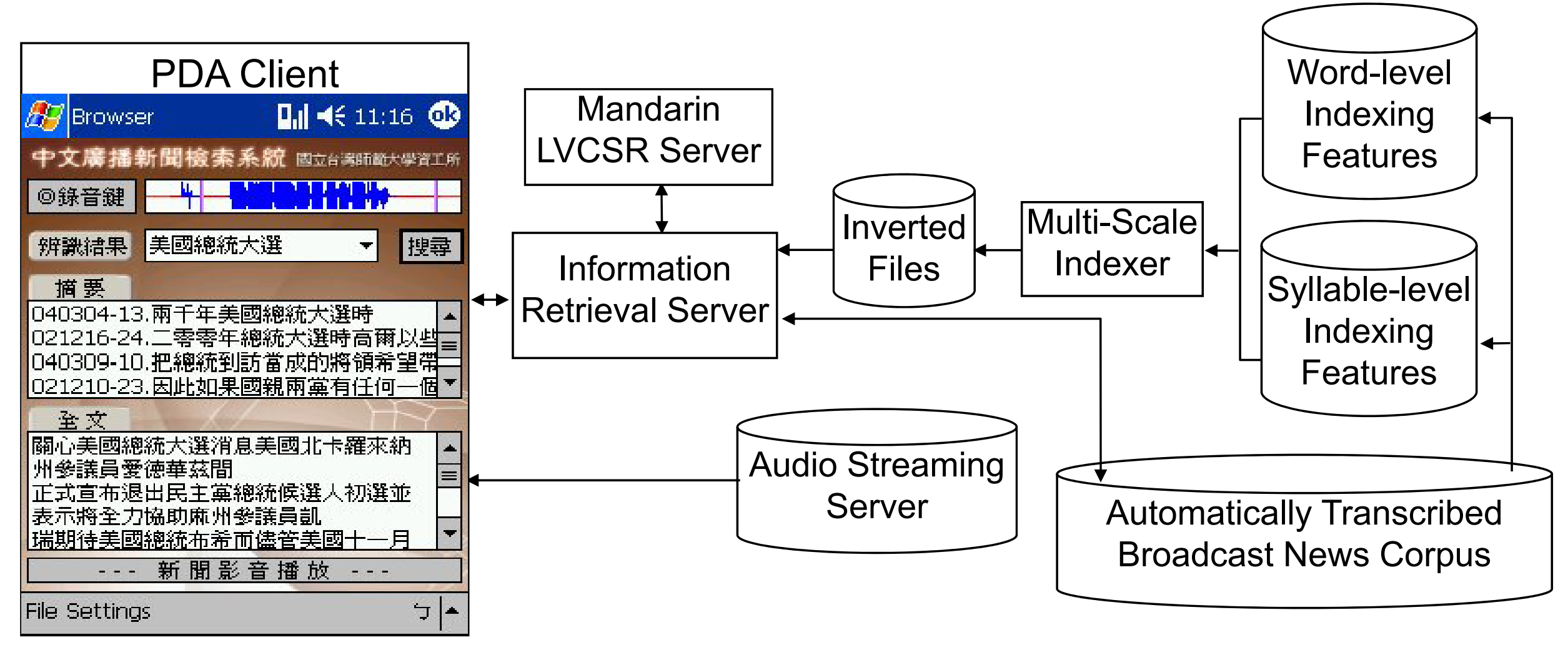

C.f. B. Chen, Y..T. Chen, C.H. Chang, H.B. Chen, "Speech Retrieval of Mandarin Broadcast News via Mobile Devices," Interspeech2005 


\section{Speech-based Information Retrieval (6/6)}

- PDA-based IR system for digital archives

- Current deployed at National Museum of History, Taipei
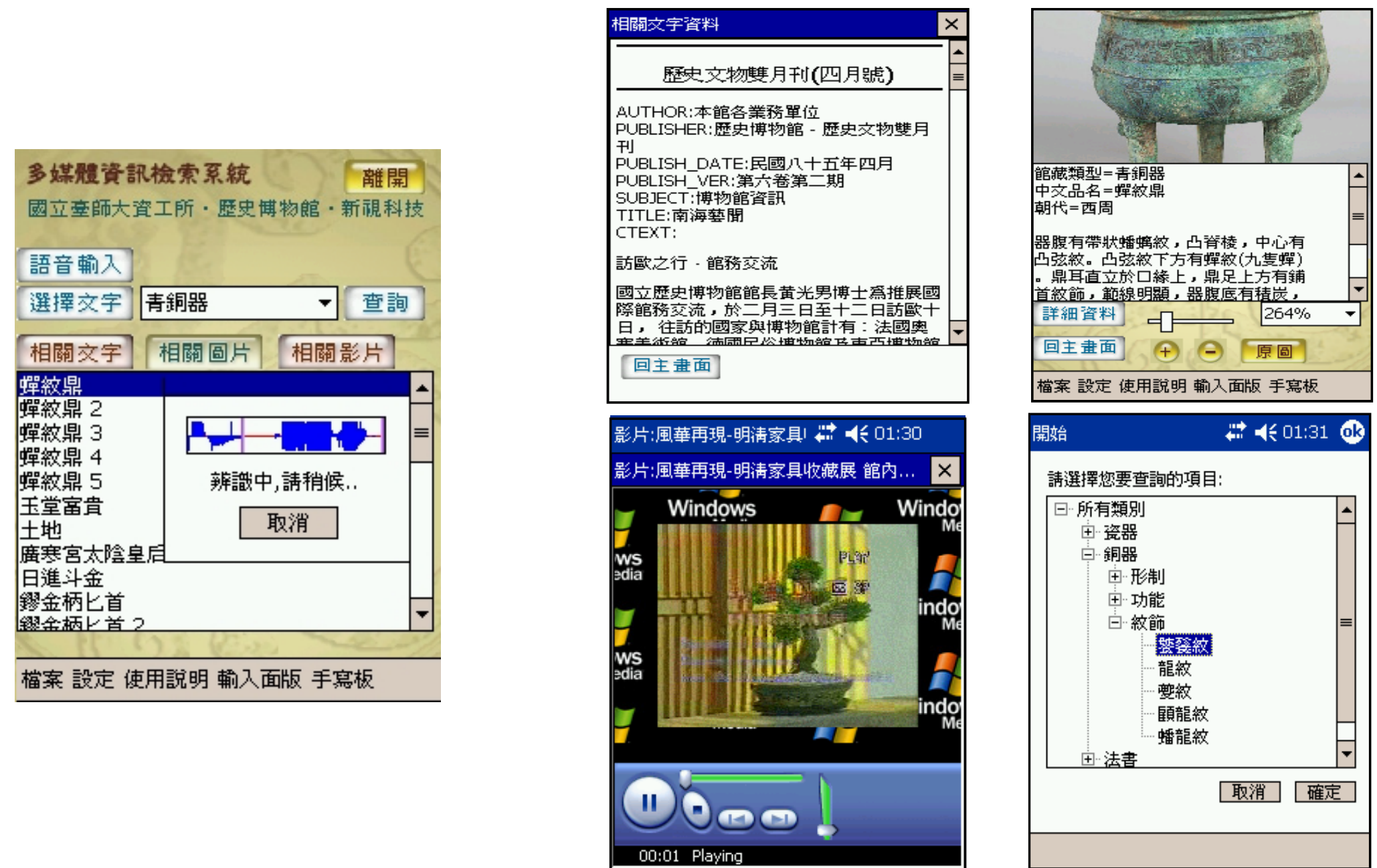


\section{Spoken Document Organization and Understanding (1/2)}

- Problems

- The content of multimedia documents very often described by the associated speech information

- Unlike text documents with paragraphs/titles easy to look through at a glance, multimedia/spoken documents are unstructured and difficult to retrieve/browse

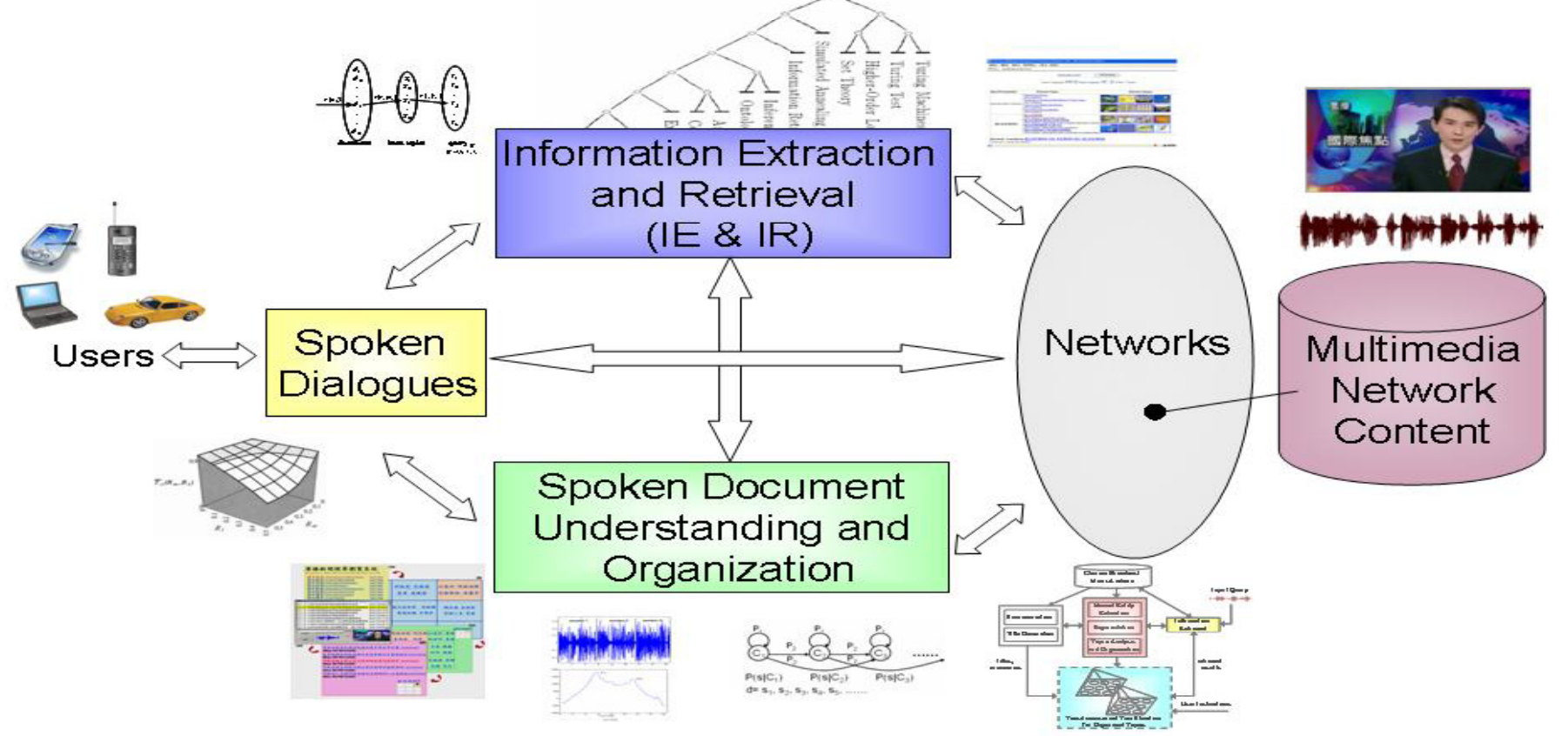

C.f. L.S. Lee and B. Chen, "Spoken document understanding and organization," IEEE Signal Processing 


\section{Spoken Document Organization and Understanding (2/2)}

- For example, spoken documents can be clustered by the latent topics and organized in a two-dimensional tree structure, or a two-layer map

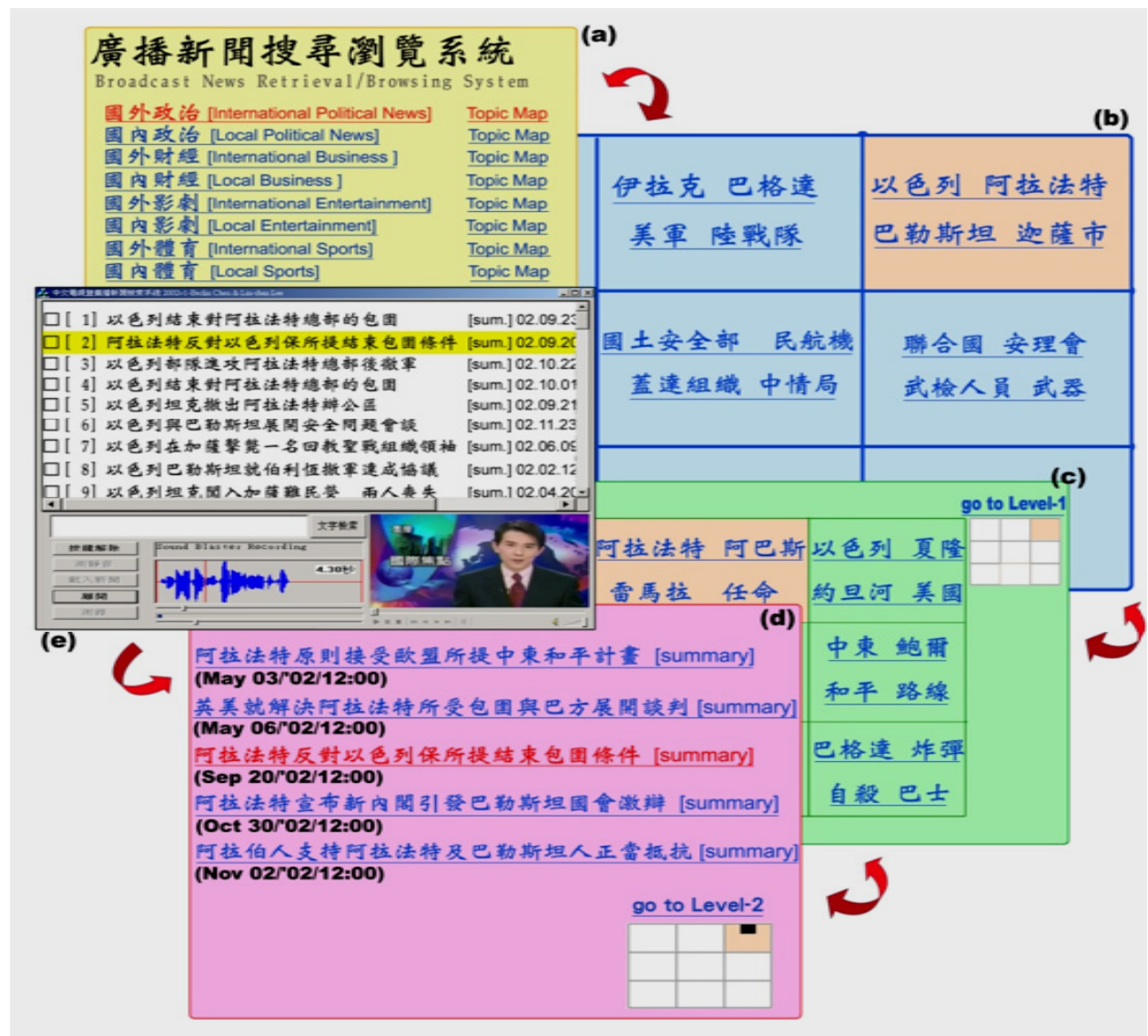

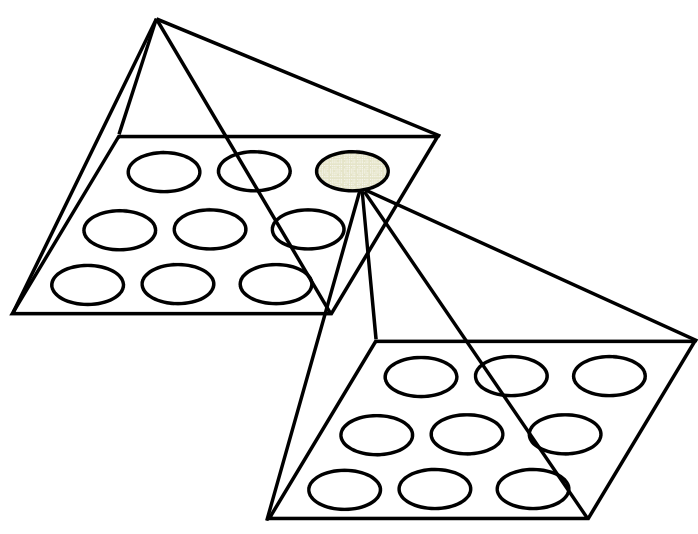

Two-dimensional

Tree Structure for Organized Topics 


\section{Speech-to-Speech Translation}

- Multilingual interactive speech translation

- Aims at the achievement of a communication system for precise recognition and translation of spoken utterances for several conversational topics and environments by using human language knowledge synthetically (adopted form ATR-SLT )

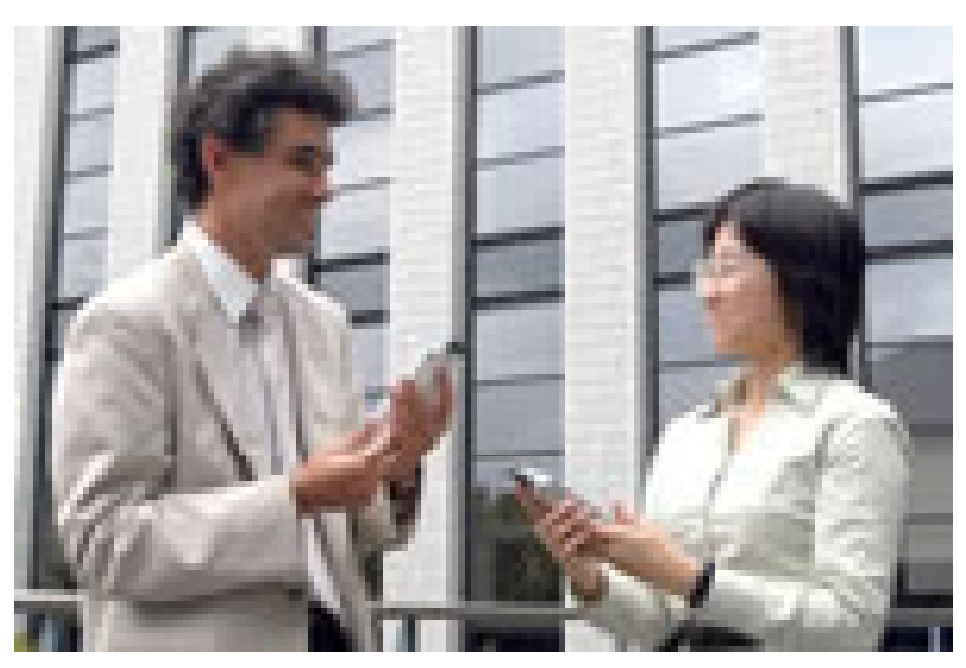

ATR-SLT

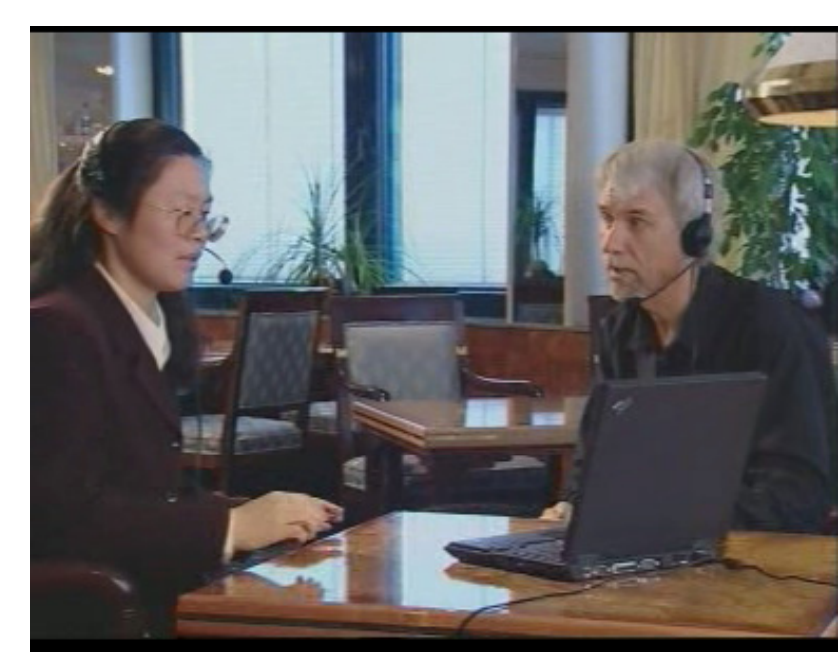

IBM Mastor Project 


\section{Map of Speech Processing Research Areas}

Applications

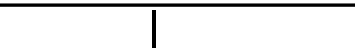

Applied

Technologies
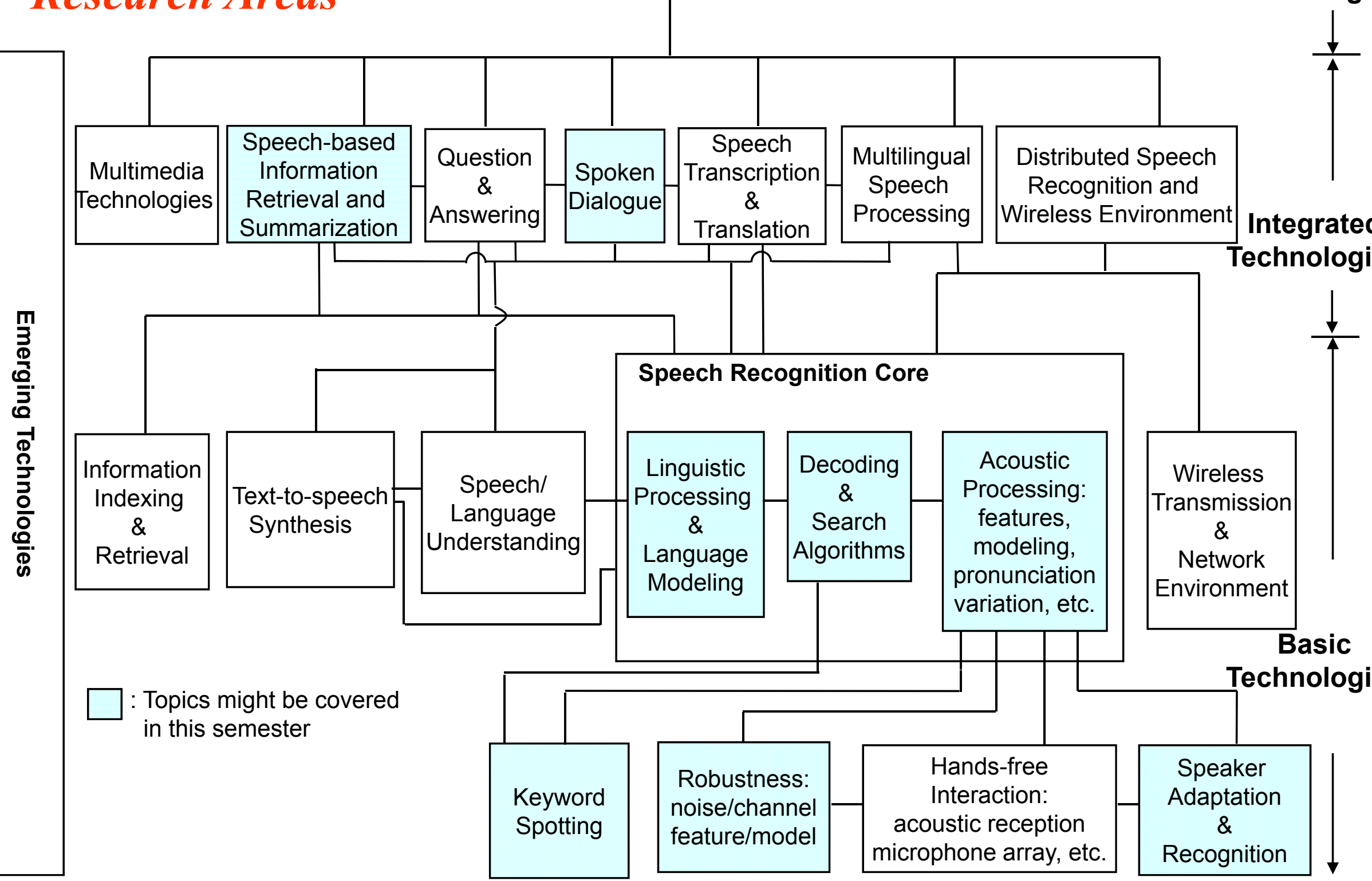

Topics might be covered in this semester

Adapted from Prof. Lin-shan Lee
Integrated Technologies
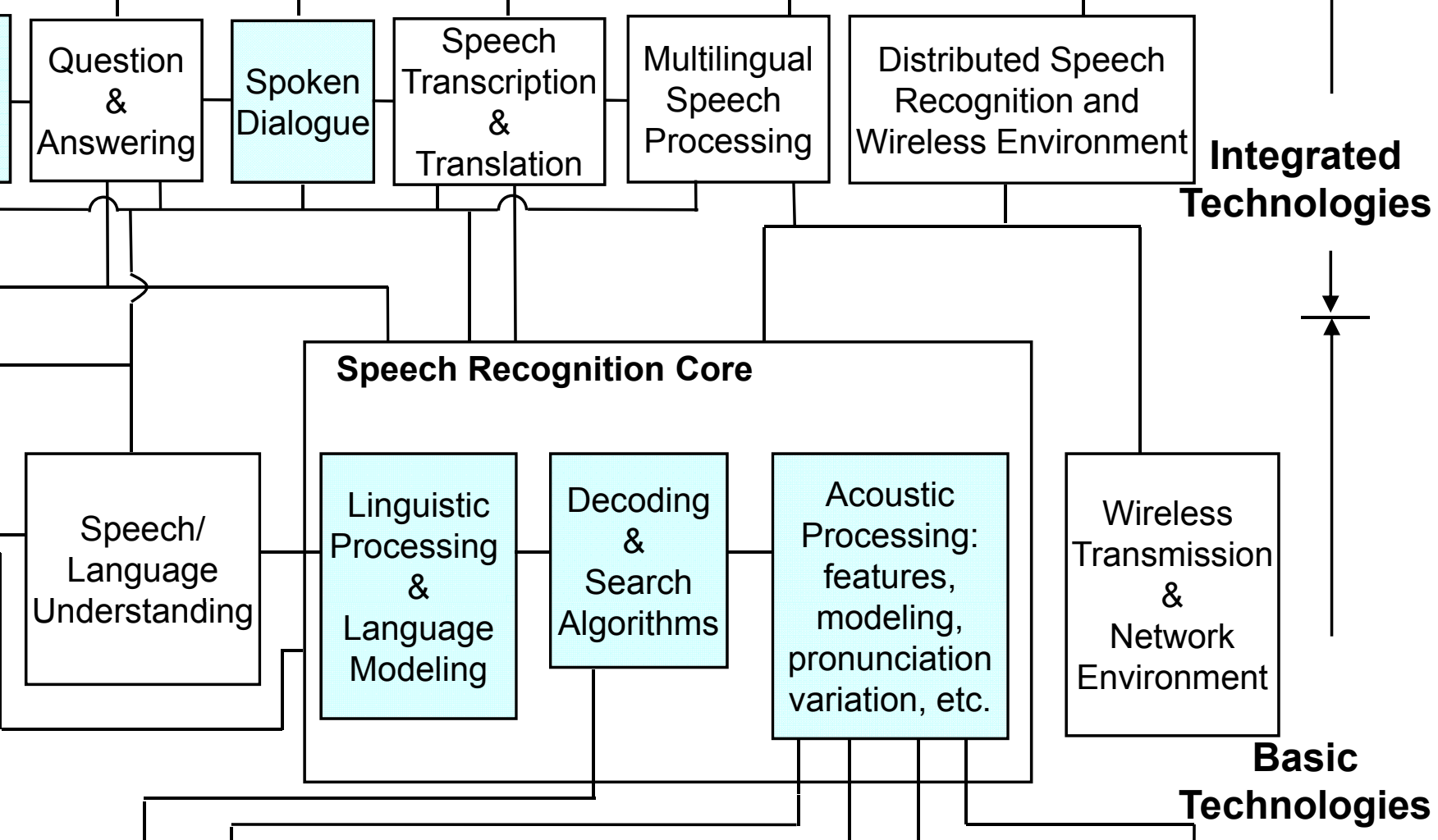

Speaker Adaptation

\&

Recognition
Wireless

Transmission

\&

Network

Environment

Basic Technologies 


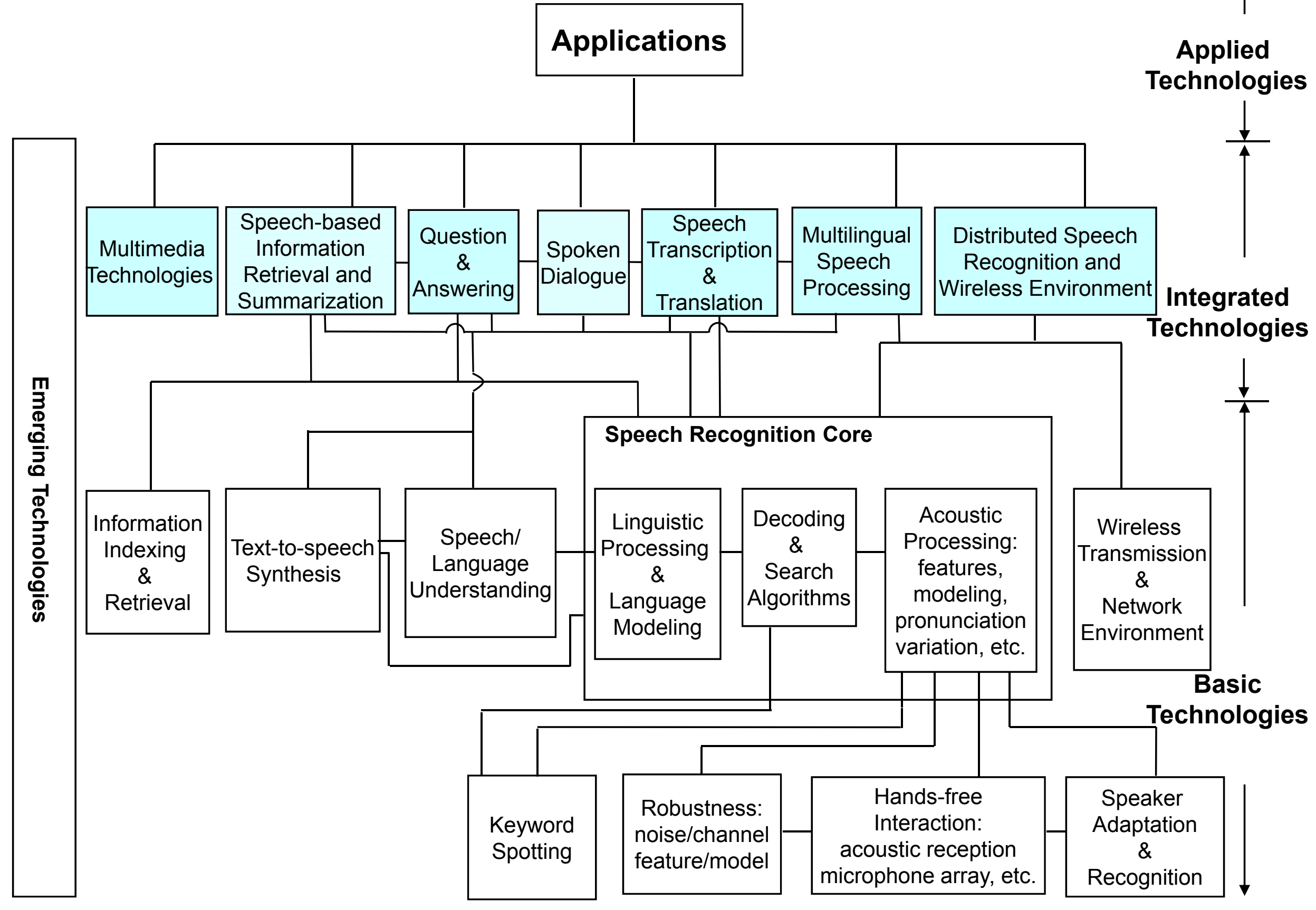




\section{Different Academic Disciplines}

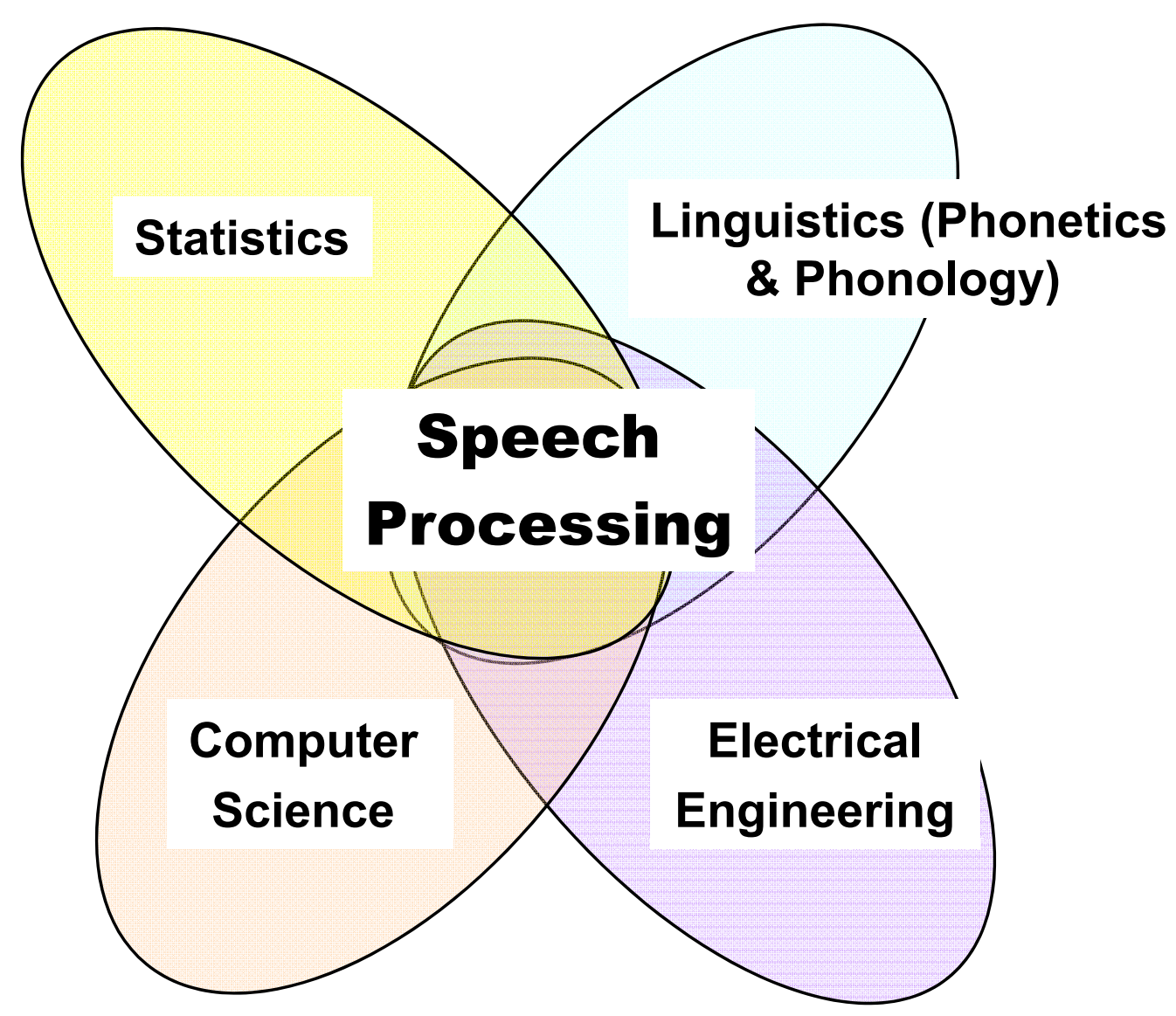




\section{Speech Processing Toolkit (1/2)}

- HTK (Hidden Markov Model ToolKit)

- A toolkit for building Hidden Markov Models (HMMs)

- The HMM can be used to model any time series and the core of HTK is similarly general-purpose

- In particular, for the acoustic feature extraction, HMMbased acoustic model training and HMM network decoding 


\section{Speech Processing Toolkit (2/2)}

- HTK (Hidden Markov Model ToolKit)

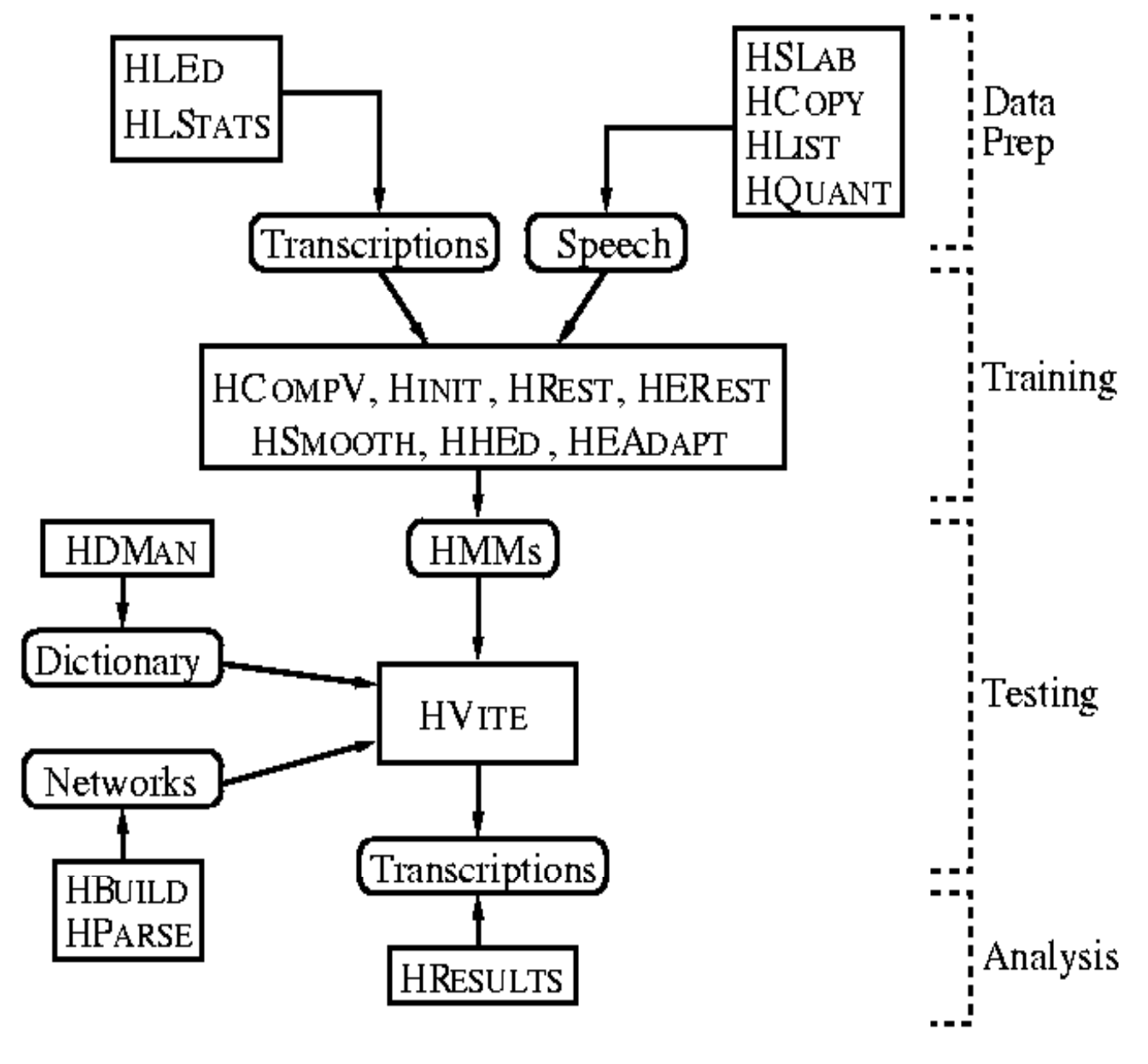




\section{Speech Industry (1/3)}

- Telecommunication

- Information Appliance

- Interactive Voice Response

- Voice Portal

- Multimedia Database

- Education

- $\ldots . .$.
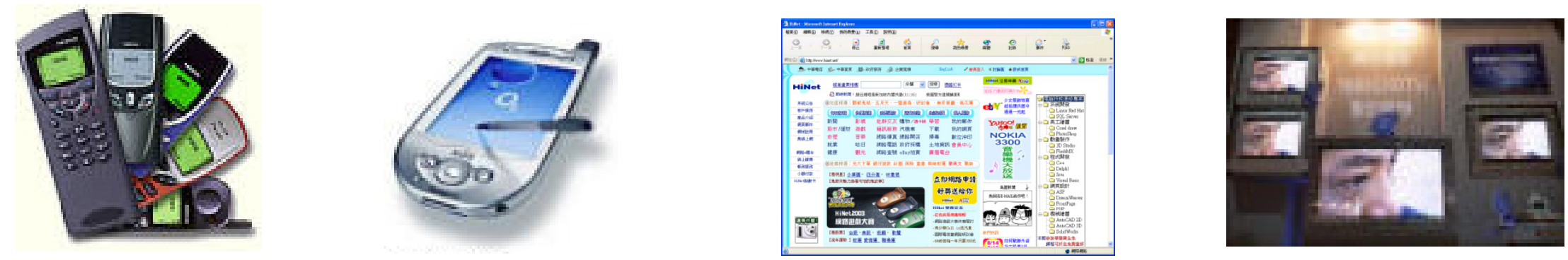


\section{Speech Industry (2/3)}

- Microsoft: Smart Device/Natural UI

使用介面的發展

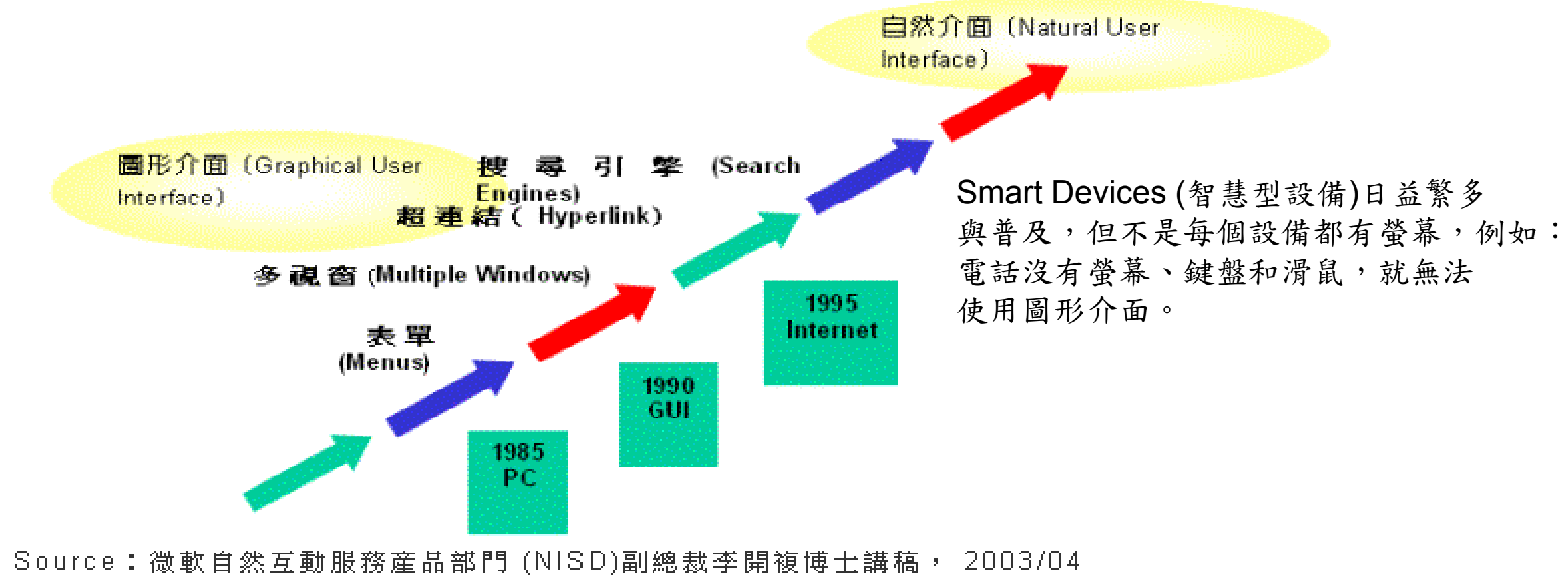

.NET 的最初構想, 以符合人類需求的自然介面, 其包括一

一語音合成

一語音辨識技術

- 結合XML為基礎的網路服務 


\section{Speech Industry (3/3)}

- Microsoft: Smart Device/Natural UI

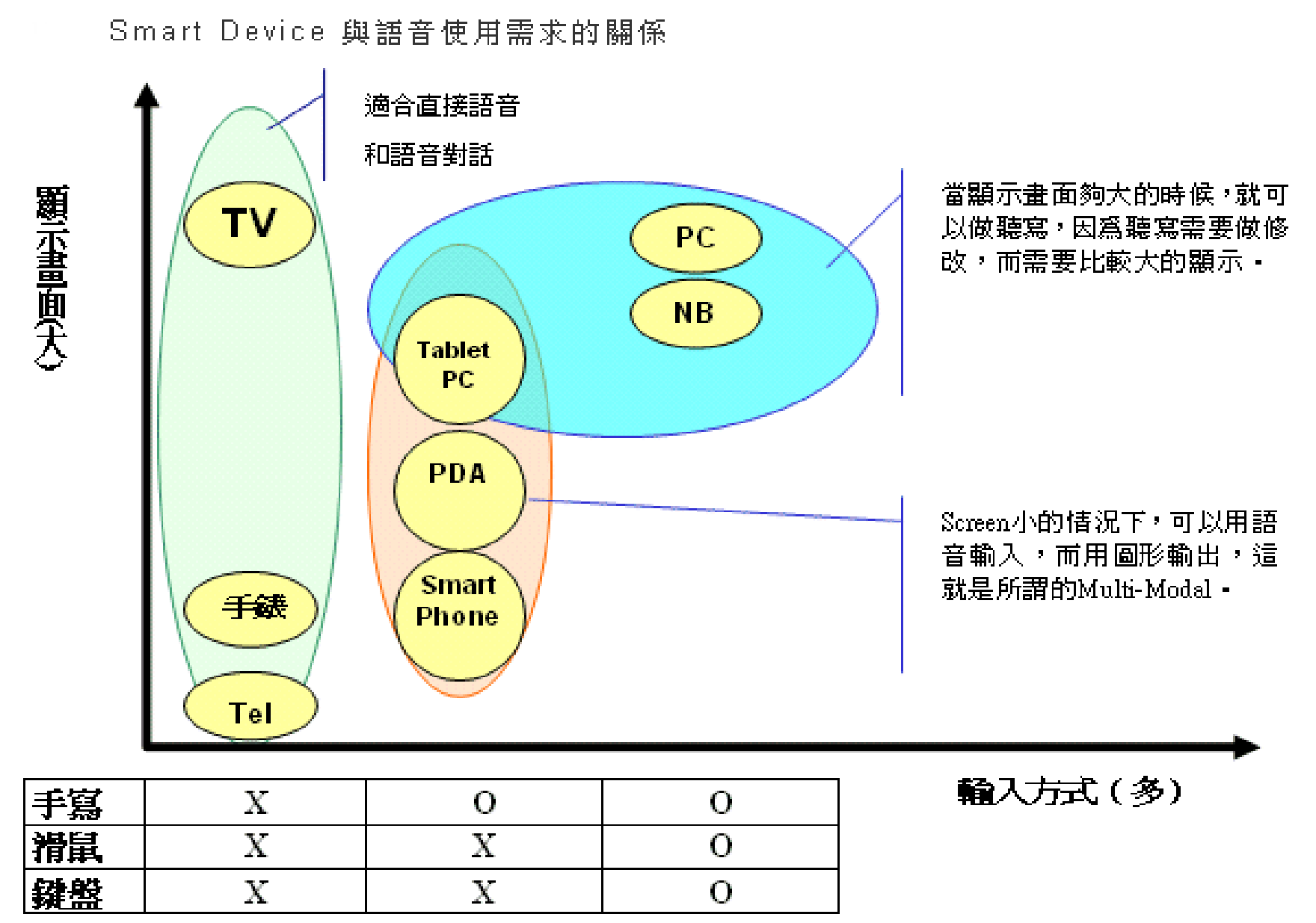




\section{Journals \& Conferences}

- Journals

- IEEE Transactions on Audio, Speech and Language Processing

- Computer Speech \& Language

- Speech Communication

- Proceedings of the IEEE

- IEEE Signal Processing Magazine

- ACM Transactions on Speech and Language Processing

- ACM Transactions on Asian Language Information Processing

$-\ldots$

\section{- Conferences}

- IEEE Int. Conf. Acoustics, Speech, Signal processing (ICASSP)

- Annual Conference of the International Speech Communication Association (Interspeech)

- IEEE Workshop on Automatic Speech Recognition and Understanding (ASRU)

- International Symposium on Chinese Spoken Language Processing (ISCSLP)

- ROCLING Conference on Computational Linguistics and Speech Processing

$-\ldots$ 


\section{Tentative Schedule}

\begin{tabular}{|l|l|}
\hline Date & \multicolumn{1}{|c|}{ Topic List } \\
\hline $03 / 27$ & Overview \& Introduction \\
\hline & Hidden Markov Models \\
\hline & Spoken Language Structure \\
\hline & Acoustic Modeling \& HTK Toolkit \\
\hline & Statistical Language Modeling \& SRI LM Toolkit \\
\hline & Midterm \\
\hline & Speech Signal Representations \\
\hline & Digit Recognition, Word Recognition and Keyword Spotting \\
\hline & Large Vocabulary Continuous Speech Recognition \\
\hline & Speech Enhancement and Robustness \\
\hline & Model Training and Adaptation Techniques \\
\hline & Utterance Verification and Confidence Measures \\
\hline & FINAL/Project \\
\hline
\end{tabular}

